\title{
Pneumatic Quasi-Passive Actuation for Soft Assistive Lower Limbs Exoskeleton
}

\begin{abstract}
Christian Di Natali ${ }^{1 *}$, Ali Sadeghi ${ }^{2}$, Alessio Mondini ${ }^{3}$, Eliza Bottenberg ${ }^{4}$, Bernard Hartigan ${ }^{5}$, Adam De Eyto ${ }^{5}$, Leonard O'Sullivan ${ }^{5}$, Eduardo Rocon ${ }^{6}$, Konrad Stadler ${ }^{7}$, Barbara Mazzolai ${ }^{3}$, Darwin G. Caldwell ${ }^{1}$ and Jesús Ortiz ${ }^{1}$

${ }^{1}$ XoLab, Department of ADVR-IIT Advanced Robotics, Istituto Italiano di Tecnologia, Genoa, Italy, ${ }^{2}$ Department of Biomechanical Engineering, University of Twente, Enschede, Netherlands, ${ }^{3}$ Department of CMBR-IIT Center for Micro-BioRobotics, Istituto Italiano di Tecnologia, Pontedera, Italy, ${ }^{4}$ Smart Functional Materials Research Group, Saxion University of Applied Sciences, Enschede, Netherlands, ${ }^{5}$ Design Factors Group, University of Limerick, Limerick, Ireland, ${ }^{6}$ Consejo Superior de Investigaciones Cientificas (CSIC), Madrid, Spain, ${ }^{7}$ Institute of Mechatronic Systems, ZHAW Zurich University of Applied Sciences, Winterthur, Switzerland
\end{abstract}

There is a growing international interest in developing soft wearable robotic devices to improve mobility and daily life autonomy as well as for rehabilitation purposes. Usability, comfort and acceptance of such devices will affect their uptakes in mainstream daily life. The XoSoft EU project developed a modular soft lower-limb exoskeleton to assist people with low mobility impairments. This paper presents the bio-inspired design of a soft, modular exoskeleton for lower limb assistance based on pneumatic quasi-passive actuation. The design of a modular reconfigurable prototype and its performance are presented. This actuation centers on an active mechanical element to modulate the assistance generated by a traditional passive component, in this case an elastic belt. This study assesses the feasibility of this type of assistive device by evaluating the energetic outcomes on a healthy subject during a walking task. Human-exoskeleton interaction in relation to task-based biological power assistance and kinematics variations of the gait are evaluated. The resultant assistance, in terms of overall power ratio $(\Lambda)$ between the exoskeleton and the assisted joint, was $26.6 \%$ for hip actuation, $9.3 \%$ for the knee and $12.6 \%$ for the ankle. The released maximum power supplied on each articulation, was $113.6 \%$ for the hip, $93.2 \%$ for the knee, and $150.8 \%$ for the ankle.

Correspondence:

Christian Di Natal christian.dinatali@iit.it

Received: 19 September 2019 Accepted: 06 May 2020 Published: 30 June 2020

Citation: Di Natali C, Sadeghi A, Mondini A, Bottenberg E, Hartigan B, De Eyto A, O'Sullivan L, Rocon E, Stadler K, Mazzolai B, Caldwell DG and Ortiz J (2020) Pneumatic Quasi-Passive Actuation for Soft Assistive Lower Limbs Exoskeleton. Front. Neurorobot. 14:31. doi: 10.3389/fnbot.2020.00031
Keywords: soft exoskeleton, exosuit, robotic wearable device, quasi-passive actuation, legged locomotion, gait assistance

\section{INTRODUCTION}

There is a growing interest across several fields in the use of wearable sensors and robotic technologies, including exoskeletons. To date, the most common field where such wearable devices have been applied is in rehabilitation. Examples of stationary (non-user grounded) exoskeletons include the Lokomat (Jezernik et al., 2003) and LOPES (Veneman et al., 2007). Both are used in clinical settings with the patient walking on a treadmill. Traditionally, mobile exoskeletons, or orthosis, assist paraplegic individuals when walking. They have been developed to assist with daily tasks involving movement. To restore some degree of legged motility to people with pathologies causing severe loss of mobility, rigid and bulky devices remain the conventional solution. Examples 
of lower limb exoskeletons are given by Farris et al. (2011) and Murray et al. (2014), where parallel robotic legs connect the users' waist and feet through kinematic chains.

When targeting assistance at people with moderate to low impairments, a further level of simplification in the device can be adopted. Examples are given by navigation assistance devices developed to address the needs of the elderly, as in Kong and Jeon (2006) and Ikehara et al. (2011), where slim designs are presented, but traditional mechanical transmission mechanisms are still employed resulting in heavy solutions.

Since the next generation of exoskeletons should address not only the degree of assistance, but also the usability/acceptance by end users, there has been an evolution from exoskeletons to exosuits. Exosuits, introduced in Awad et al. (2017), Jin et al. (2017), and Schmidt et al. (2017), have ankles, knees or hips that are supported by a soft or hybrid structure and an active tendon driven actuation system. These examples benefit from a soft wearable structure, which helps to reduce the burden of the device. On the other hand, issues (e.g., weight, power consumption, and a cumbersome design due to the inclusion batteries and other mechanical components) can arise from using active electrical motors combined with a cable or belt to actively assist joints.

The presented trend underlines the desire to avoid bulky, rigid, heavy exoskeletons, by using light, soft, and shapely wearable devices. Thus, system autonomy, usability and acceptance became the foundation of the XoSoft EU project. The XoSoft EU project consortium developed a user-centered design based, soft, modular, bio-mimetic, and quasi-passive exoskeleton to assist users with low to moderate mobility impairments, such as the elderly, and post-stroke or partial spinal cord injury subjects (Power et al., 2016; Ortiz et al., 2017).

Previous work as part of this development includes the XoSoft Beta 1 prototype (Di Natali et al., 2019) and a quasi-passive actuation $(Q P A)$ strategy for system optimization introduced in Ortiz et al. (2018), where electromagnetic clutches are used to modulate the passive elements employed to store the mechanical energy. These systems do not provide any active force, but rather transfer mechanical stored energy between gait phases. To further reduce weight and size of the proposed actuation system, soft actuators have been investigated. Soft clutches should meet the following specifications: (1) linear sliding movement to be installed along the limbs; (2) able to change load bearing capabilities under certain stimuli; (3) able to vary its initial length; (4) able to respond to the imposed motion by changing from a compliant, freely elongating condition to a stiff state.

The interest in soft actuators is evident from the literature, as demonstrated by Van Ham et al. (2009), Manti et al. (2016), and Wolf et al. (2016). In particular, soft clutches are valuable to human-robot interaction and soft robotics. The ongoing research on soft actuators aims to develop clutches, brakes, dampers and devices based either on specific phase change materials (e.g., low melting point materials, Taghavi et al., 2018, electro- and magneto-rheological fluids, Petek, 1992; Carlson and Jolly, 2000; Oh and Onoda, 2002; Nikitczuk et al., 2010; Alkan et al., 2013) or on a friction-based mechanism (e.g., electro-adhesion, Diller et al., 2016; Ramachandran et al., 2019, jamming and cable tensioning Walsh et al., 2007; Van Dijk et al., 2011). Vacuumcontrolled jamming (either particles or layers) is a suitable technology that has found a number of uses within the robotics community (Brown et al., 2010; Follmer et al., 2012; Jiang et al., 2012; Stanley et al., 2013; Li et al., 2014; Zubrycki and Granosik, 2017; Sadeghi et al., 2018). In the field of wearable robotics, Hauser et al. (2017) a wearable joint support was developed based on granular jamming controlled by pressure. As highlighted in Kawamura et al. (2003), a laminated passive element could be used for rigid limb attachments (i.e., orthosis), Bureau et al. (2011) and Tonazzini et al. (2018). In wearable haptics, Mitsuda (2017) implements a force display based on fabric jamming technology, whereas layer jamming has been employed as a brake in Choi et al. (2018) and Ramachandran et al. (2019).

In Sadeghi et al. (2019a), the authors demonstrated the actuation mechanism of a textile based clutch $(T B C)$ being applied to the wearable XoSoft. The TBC stiffness increases tens of times by applying negative pressure to two parallel textiles featuring repetitive grooves, contained within an airtight deformable chamber. The layers pack together and the grooves interconnect to restrict the relative movement. This, on a microscopic scale bears some similarity to natural muscle. The $T B C$ results indicated a viable option for developing a soft controllable clutch wearable system. The braking force is determined by the friction force, which is controlled by the vacuum pressure applied between the two layers.

The major contributions of this work are the development of a new soft, wearable device, the Gamma prototype, that uses soft pneumatic QPA to deliver gait assistance. The modularity of the device is presented, and configurations for hip and knee flexion, and ankle plantar-flexion assistance are demonstrated. The system design is illustrated. The actuators are validated experimentally, followed by an assessment of the overall system to determine its effectiveness during testing on a healthy subject during the walking task. In section 2, the full design is presented. In section 3, the modeling of the exoskeleton and human interaction is addressed. In section 4 the experimental protocol and results are explained and discussed. Finally, in section 5, conclusions and future developments are addressed.

\section{DESIGN}

The Gamma prototype developed within the XoSoft project, is the most advanced iteration of the user-centered design approach. The soft exosuit is tested to assess functionality, validity and reliability. The device meets the essential requirements and is classified as a class 1 medical device compliant with IEC 60601. When compared to the previous Beta 1 variant, several issues have been addressed to improve and enhance the actual exosuit: to facilitate donning and doffing, to improve comfort and acceptability, and to test the effectiveness of use and operation with different primary users. Consequently, the Gamma prototype involved a truly modular approach, allowing actuation of different joints, unilaterally or bilaterally, depending on the users' needs. A variety of primary users were targeted to test wearing the Gamma. Thus, the current design prototype 
addressed all these features of modularity, and reconfigurability to subject needs, showing the potential of becoming a platform for rehabilitation or use as a daily assistive device.

Figure 1 shows pictures and a technical drawing of the actuator arrangement on the soft exoskeleton including: sensing/sensors, the QPA technologies, and the garment centered mechanical structure of the Gamma prototype. The main technical elements composing this rehabilitation platform are presented in Figure 2, and are summarized as follows:

- TBC: A mechanical clutch actuated by vacuum pressure to block the mechanism that allows the controlled storage and release of energy.

- Elastic Band (EB): Elastic element to store mechanical energy. On one side (distal attachment) it is connected directly to the body segment. The other side (proximal) is connected to one end of the TBC.

- Body Attachment: Body attachment required to transmit the forces from the actuators to the wearer's body.

- Shoe Sensors: Sensors placed on the plantar side of the foot to detect the contact between the foot and the floor. Used as an input for the motion/gait segmentation.

- IMU Sensors: Sensors placed on each thigh and shin to detect the knee angular displacement. Used as an input for the motion/gait segmentation.

The actuation principle is defined as Quasi-Passive Actuation and it is composed of an EB and the TBC in series. QPA refers to any controllable element that cannot apply a non-conservative motive force (Endo et al., 2006). Thus, QPA includes any combination of variable-dampers or clutches in conjunction with passive components such as springs. The work presented in Di Natali et al. (2019) on the previous soft wearable device (Beta 1 prototype), introduced the QPA unit for a unilateral hip and knee assistive configuration. The modular design description and assessment is also addressed. In particular, the Beta 1 prototype was designed and tailored for a unique patient with a lower limb mobility problem on the right side. unlike the Beta 1 prototype, the control system of the Gamma uses a biomimetic approach based on gait segmentation to appropriately activate or deactivate the QPA to alternate between the storing and releasing actuation phases. The control exploits insole sensors and IMUs feedbacks to identify gait patterns.

\subsection{Design Description}

\subsubsection{Garment}

The Gamma prototype garment was developed using feedback from the tests with the Beta 1 prototype. To define a fabric suitable for the prototype, the following properties are taken into account: fiber composition, weight, elongation/stretch, absorbency/wetting and comfort (Mecheels and Umbach, 1977). A one-size-fits-all garment was developed for all the participants by ensuring optimal fitting for the key measurements. To improve comfort and breathability, soft fabric (100\% polyester 75D Interlock fabric, 210 grams per yard) was selected for the main pants section of the garment.

Reinforced elements such as Polyester webbings and Nylon 210D have been used to enable sufficient inelastic response on the body attachments where forces will be applied. The main
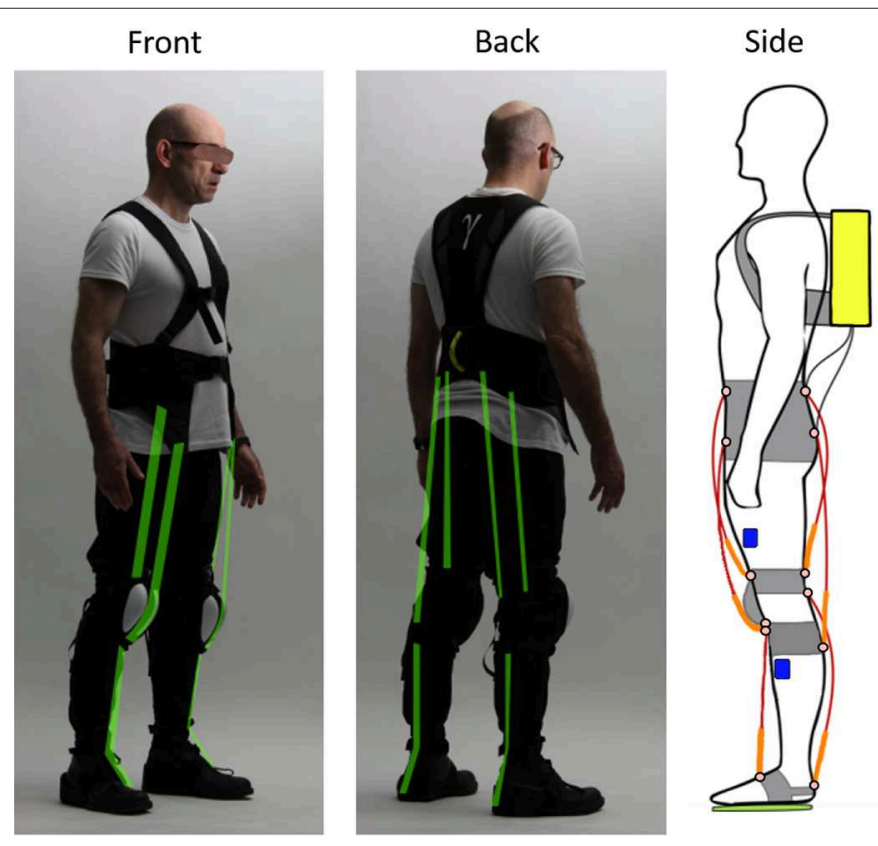

Actuation Line
TBC
EB
$\square \quad$ Attachment point

\section{Garment reinforcements}

Shoe Sensor

IMU Sensor

Backpack

FIGURE 1 | Overview of the technologies integrated in the prototype Gamma. The backpack contains: the pneumatic system, electronic boards and the central computer. The front and back views show in green, all the possible QPAs lines that can be installed on the garment. The side view displays for each actuator line the TBCs and related EBs, attachment points, shoe sensors, IMU sensors placement, garment reinforcement points, and backpack. Written informed consent was obtained from the individual pictured in the figure. 


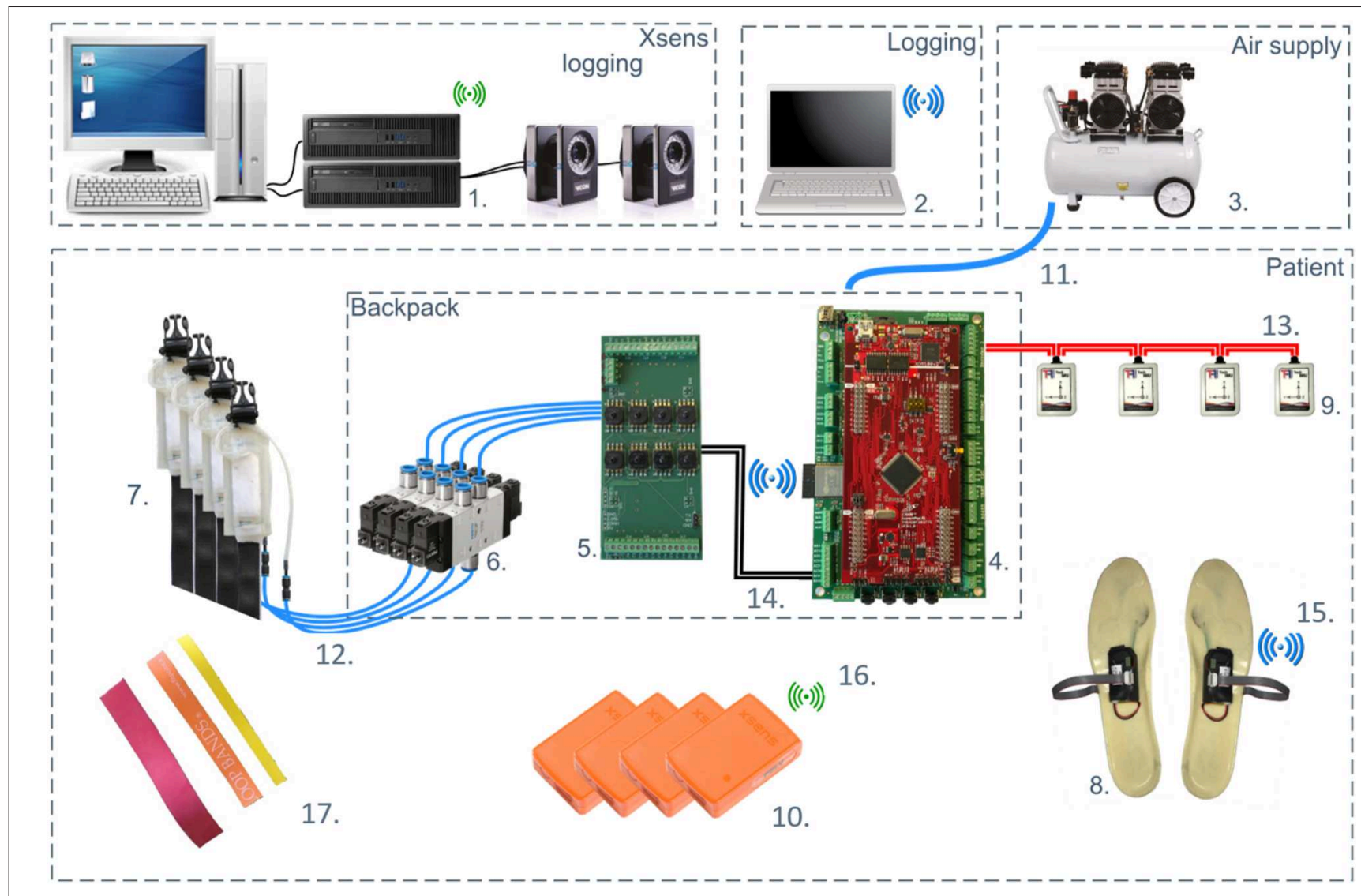

FIGURE 2 | Overview of the system components and layout for Gamma prototype version. (1) Xsens logging; (2) Exoskeleton state and sensors logging; (3) Air compressor; (4) Central processor; (5) Valve controller board; (6) Valves; (7) TBCs; (8) Shoe sensors; (9) IMUs (for control); (10) Xsens system (for motion tracking); (11) Compressed air; (12) Vacuum lines; (13) CAN bus 1; (14) CAN bus 2; (15) Wi-Fi network 1, (16) Wi-Fi network 2; (17) EBs. Main hardware components of the exoskeleton are described in section 2.1.

webbings and reinforcements are displayed in gray in Figure 1. To avoid any contact between the front knee actuation and the patella bone, a protective plastic cup is employed to rise the actuation from the knee profile (displayed on the lateral view of Figure 1). Loose and elastic fabric are used to facilitate quick donning. The overall weight of the garment is $0.8 \mathrm{~kg}$.

\subsubsection{Actuation Units}

As previously mentioned, the actuation is defined as quasipassive since the system stores energy from the user during certain gait phases delivering it when needed. The actuation unit is composed of a EB and pneumatic clutch $(T B C)$ in series. The EB and TBC assembly is able to modulate the assistance to the user, by controlling the storing and releasing phases of the mechanical power. If the clutch is not engaged, the actuation line results are neutral to the user, as no storing or releasing phase is involved, while the user is moving. The TBC is an assembly of two inextensible webbings equipped with an array of $2.3 \mathrm{~mm}$ wide parallel rigid bumps (similar to a rack gear) fabricated directly on them by a hot embossing technique, and two elastic bands. Internally, within the same envelop, the recall elastic element is connected to both parallel layers. These elastic bands have an intrinsic elasticity which creates a small constant stiffness. This ensure that the TBC-EB unit is always under small tension during movement, remaining tight against the limbs. The complete unit is air sealed by a silicone elastomer cover (Sadeghi et al., 2019b). When the vacuum pressure is applied, the TBC system is engaged, with the $T B C$ stiffness characteristic changing from elastic to rigid along the pulling direction. When the clutch is engaged, it becomes stiff and the elastic band starts elongating as the user is moving the joint.

From an implementation standpoint, and based on previous studies and simulations by Ortiz et al. (2018) and Di Natali et al. (2019), it is possible to characterize each specific actuation line for the hip, knee and ankle joints by maximum length, elongation, relative angular displacement and predicted force provided by the actuation. When the clutch is disengaged, unappreciated force is exerted by the system to the user and this configuration is defined transparent.

The initial length and maximum elongation associated with each actuation segment are measured on an average size subject as defined by Dempster and Gaughran (1967). These data are provided in Table 1 and the $T B C$ geometry is displayed in Figure 3A. In Table 1, "max travel" represents the maximum elongation permitted by the TBC if actuation is not needed and the system has to be transparent to the user. Based on 
the information in Table $\mathbf{1}$, two families of clutches have been developed. The first family is used for the upper leg (hip and knee flexion-extension) and the second family for both ankle plantarflexion and dorsiflexion. Table 2 provides the TBC characteristics. Each clutch has a webbing loop on one side for quick installation as shown in Figure 3A. The webbing loops on both sides of the actuation line connect it to the garment using adjustable dog-bone anchors. The TBC terminates with approximately $200 \mathrm{~mm}$ long webbing that connect to a cam

TABLE 1 | Geometrical characteristics collected to guide the TBC and EB design.

\begin{tabular}{lcccc}
\hline Joint & $\begin{array}{c}\text { Max force } \\
{[\mathbf{N}]}\end{array}$ & $\begin{array}{c}\text { Actuation } \\
\text { line length }\end{array}$ & $\begin{array}{c}\text { TBC travel } \\
\text { (max travel) } \\
\text { [mm] }\end{array}$ & $\begin{array}{c}\text { Expected } \\
\text { elastic } \\
\text { elongation } \\
\text { [mm] }\end{array}$ \\
\hline Hip extension & 30 & $200-250$ & $50(100)$ & 50 \\
Hip flexion & 30 & $300-400$ & $50(100)$ & 50 \\
Knee extension & 30 & $250-350$ & $50(100)$ & 50 \\
Knee flexion & 30 & $200-300$ & $50(100)$ & 50 \\
Ankle dorsiflexion & 20 & $220-280$ & $20(40)$ & 20 \\
Ankle & 60 & $250-300$ & $20(50)$ & 30 \\
plantarflexion & & & &
\end{tabular}

buckle, and then to the EB, (Figure 3B). This set-up permits adjustments of the distance between TBC and corresponding EB. The EBs used in the Gamma prototype are latex-made elastic bands connected to the webbing material through a vulcanization process. These are displayed in Figure 3B. Table 3 shows the EB's design characteristics, based on the assistance required. The EBs stiffness characteristics are reported in Table 4, as a third order function of elongation percentage as in Equation (1) (the elasticity trend is displayed in Figure 3C)

$$
f_{k}\left(\alpha_{k}\right)=a_{3} \Delta L_{k}^{3}+a_{2} \Delta L_{k}^{2}+a_{1} \Delta L_{k}
$$

where $\Delta L_{k}$ is the percentage of elongation.

\begin{tabular}{lccccc}
\hline TABLE 2 & TBCs design & characteristics. & & & \\
\hline $\begin{array}{l}\text { TBC } \\
\text { family }\end{array}$ & $\begin{array}{c}\text { TBC } L_{0}(B) \\
{[\mathrm{mm}]}\end{array}$ & $\begin{array}{c}\text { Length of } \\
\text { interlocking } \\
\text { zone }(\mathbf{A})\end{array}$ & $\begin{array}{c}\text { Requested } \\
\text { resistance } \\
\text { force }[\mathbf{N}]\end{array}$ & $\begin{array}{c}\text { TBC travel } \\
\text { (max travel) } \\
{[\mathbf{m m}]}\end{array}$ & $\begin{array}{c}\text { Pressure } \\
{[\mathrm{bar}]}\end{array}$ \\
\hline $\begin{array}{l}\text { Upper } \\
\text { leg } \\
\text { Lower }\end{array}$ & 140 & 110 & 100 & $50(100)$ & 0.3 \\
leg & 110 & 80 & 200 & $20(50)$ & 0.3 \\
\hline
\end{tabular}

Length of interlocking zone (A) and initial length (B) are related with the figure displayed in Figure 3 A.

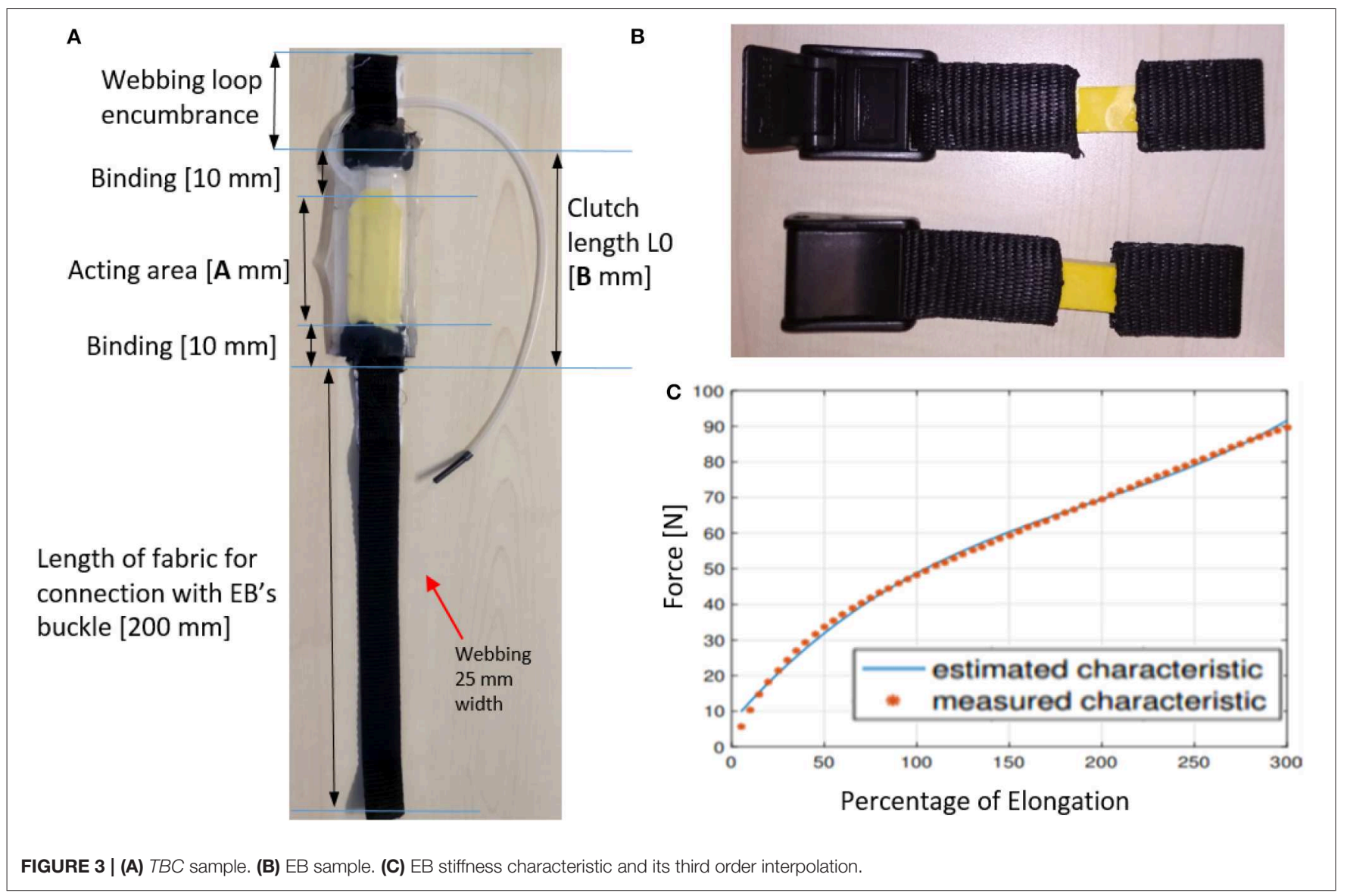


The garment has webbing anchor straps (shown in Figure 1) providing the body attachments for proximal and distal ends of the actuation lines. The waistcoat provides the body attachments for frontal and rear knee and hip actuations. At the knees, two webbings loops for body attachment are positioned, one below and one above the joint. For the distal attachment of ankle actuation, straps are worn over regular shoes providing attachment points on the front and back of the foot. The $T B C$ s are connected to the garment through webbing-loops and dog-bones.

TABLE 3 | EBs design characteristics.

\begin{tabular}{lcccc}
\hline EB family & EB $L_{0}[\mathrm{~mm}]$ & $\begin{array}{c}\text { System } \\
\text { length }[\mathrm{mm}]\end{array}$ & $\begin{array}{c}\text { Nominal } \\
\text { Stiffness } \\
{[\mathbf{N} / \%]}\end{array}$ & EB typology \\
\hline Hip extension & 20 & 100 & 0.5 & $\mathrm{~A}$ \\
Hip flexion & 50 & 150 & 0.8 & $\mathrm{~B}$ \\
Knee extension & 30 & 150 & 1 & $\mathrm{C}$ \\
Knee flexion & 25 & 120 & 0.5 & $\mathrm{~A}$ \\
Ankle dorsiflexion & 10 & 100 & 0.25 & $\mathrm{D}$ \\
$\begin{array}{l}\text { Ankle } \\
\text { plantarflexion }\end{array}$ & 20 & 140 & 10.5 & $\mathrm{~A}$ \\
\hline
\end{tabular}

TABLE 4 | EBs typology.

\begin{tabular}{lcccc}
\hline EB typology & Nominal stiffness [N/\%] & $\mathbf{a}_{\mathbf{3}}$ & $\mathbf{a}_{\mathbf{2}}$ & $\mathbf{a}_{\mathbf{1}}$ \\
\hline A & 0.5 & $3.66 * 10^{-6}$ & -0.002 & 0.565 \\
B & 0.8 & $9.06 * 10^{-6}$ & -0.005 & 1.194 \\
C & 1 & $1.23 * 10^{-5}$ & -0.007 & 1.621 \\
D & 0.25 & $2.04 * 10^{-6}$ & -0.001 & 0.312 \\
\hline
\end{tabular}

EB's stiffness as a third order polynomial fitting coefficient as in Equation (1).

\subsubsection{Pneumatic System}

The $T B C$ is activated pneumatically by applying negative pressure. TBCs are managed by means of pressure sensors and solenoid valves to connect the $T B C$ to the vacuum line, atmospheric pressure and a closed state. The closed state is provided to allow the $T B C$ to maintain the set pressure (idle state). The primary source of the pneumatic system is a compressor or air line (if present in the clinic). The pneumatic control system schematic for the exoskeleton is shown in Figure 4. The pressure regulator sets the low pressure supply (less than 6 bar) to the exoskeleton's pneumatic line. A 2-way valve (MHJ10-S-2,5-QS-6-HF, electro-valve, Festo Inc., Germany), represented by $\mathrm{A}$ in Figure 4, is connected in line with a vacuum generator to provide a vacuum when needed. The vacuum generator (VN-20-H-T6-PQ4-VQ5RO2, vacuum generator, Festo Inc., Germany), which uses the Venturi principle, can generate vacuum pressures up to $-90 \mathrm{kPa}$ with an air flow of $100 \mathrm{~L} / \mathrm{min}$. A double silencer ensures that the noise is under $50 \mathrm{~dB}$. The vacuum generator is connected to the $T B C$ through two series connected of 3-way solenoid valves (MHE2-MS1H-3/2G-QS4-K and MHE2-MS1H-3/2O-QS-4-K, electro-valve, Festo Inc., Germany). The first valve, in Figure 4, represented by $\mathrm{B}$ activates if the clutch needs to be engaged. The second 3-way valve (represented by C) connects the TBC to the external environmental pressure. If both the 3-way valves are off, the $T B C$ is isolated and the internal pressure is maintained. The opening and closing of valves is controlled by a pressure sensor (MPXV6115V, Absolute Pressure Sensor, Freescale Semiconductor, pressure range -115 to $0 \mathrm{kPa}$ ). To control multiple actuators in parallel, a multi-way manifold is placed between the pressure regulator and the vacuum generator. The Valve Driver Board is a custom design based on a Texas Instruments microcontroller (TMS320F28035) with a CAN transceiver to receive commands and return status, log and pressure sensor values.

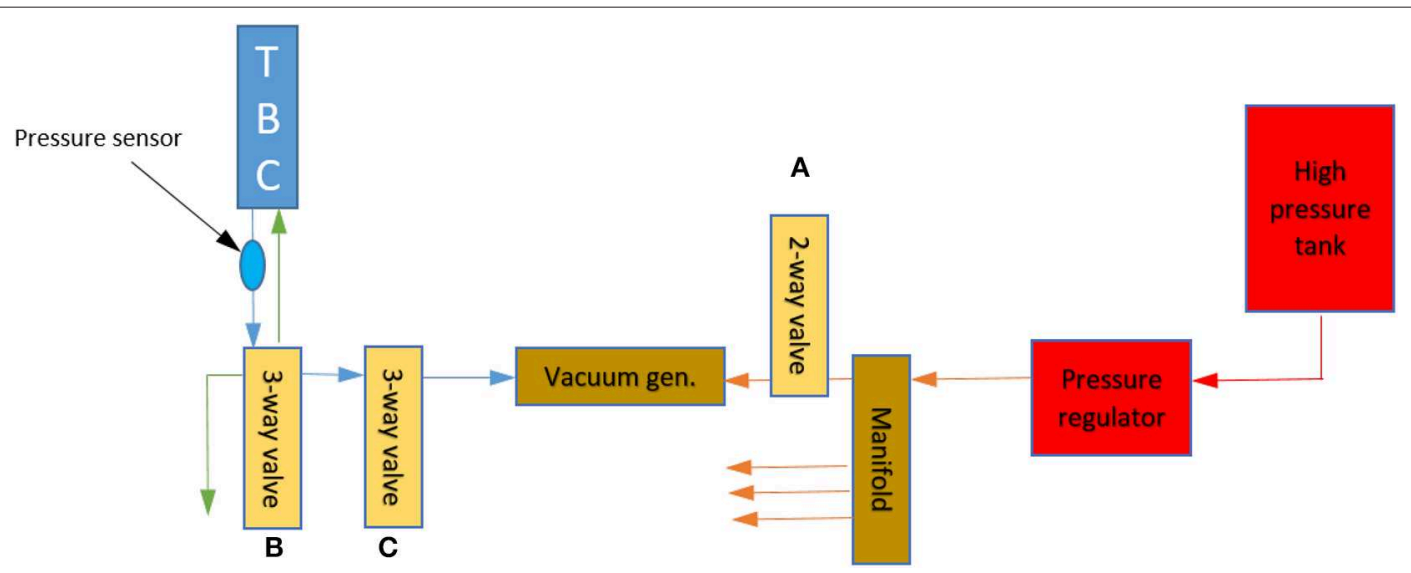

FIGURE 4 | Pneumatic circuit for TBC control. The system pressure is represented with a color map. The green arrow is for pressure of $101 \mathrm{kPa}$, blue is pressures up to $10 \mathrm{kPa}$ and below $101 \mathrm{kPa}$. The orange represents an intermediate pressure up to 6 bar (600 kPa), and the red represents the high line pressure of between 600 and $1,200 \mathrm{kPa}$. 


\subsubsection{Sensors}

A pair of custom made, soft foam (Hekapur PU, Exact Plastics, Bröckel, Germany) insoles with four embedded force sensing resistors (FSR, 1-Inch ShuntMode, Sensitronics, Bow, USA) are used to detect foot contact with the ground. There is one sensor under the heel, two under the metatarsal area and one under the phalanges (Figure 2). The pressure sensors, operating as switches provide a digital signal depending on whether a pressure threshold is exceeded. As shown in Figure 1, four IMUs (TechMCS V4, Techaid S.L., Madrid, Spain) are placed at the shin and thigh of each leg. The signal information is processed to extract the knee joint angular displacement (Cooper et al., 2009). The method directly uses the orientation matrices provided by these sensors. By fusing the sensor outputs from the ground contacts and knee angles [heel pressure signal (HS), outside insole pressure signal (OS), inside insole pressure signal (IS), toe pressure signal (TS), and the derivative of the knee angular displacement (KAD)], in real-time, the system segments the gait cycle to identify different events such as: heel strike, flat foot, front foot, toe off, positive speed inflection and negative speed inflection. Using this sensory data and sequencing, the specific control signals can be applied to provide the required assistance and actuator action (Figure 6).

\subsubsection{Electronics and Communication}

The electronics consists of two Wi-Fi modules (ESP32, Espressif, Shangai, China), for insole sensor readings, transmitting to the main central processing unit (CPU) board (Launchpad XL Development Kit, Texas Instruments, Dallas, US) with a custom extension cape board providing Power Management, I/Os and CAN Communication as shown in Figure 2. The IMU sensors communicate with the CPU through CAN bus communication. The CPU has all the peripherals needed to run all the communication; the firmware has been developed to be able to link with the Control Valve Board. The Power Supply Board and Valve Driver Board act as a decoupling interface to separate the low power signals used by the CPU from the high power signals to the valves and for CAN communication. The form factor of this board allows boards to be stacked below (Power Supply) and above (Valve Driver), to create a modular and easily replaceable system. The Power Supply board is a custom board providing enhanced $\mathrm{I} / \mathrm{O}$ and communication capabilities. It has CAN transceivers and Wi-Fi modules. Each ESP32 Wi-Fi module sends the FSR data wirelessly to the CPU. The board provides control signals and power to all the pneumatic valves (both 2-way and 3-way) using a power MOSFET and a freewheeling diode. The microcontroller draws energy from the Texas Instruments LaunchPad XL board. A 3way connector links the pin of the CAN transceiver available on the LaunchPad, providing a unique way to assemble the two boards together. The pressure sensors are sensitive to negative pressure only, maximum $-15 \mathrm{kPa}$. Each sensor can be easily plugged into the corresponding vacuum line for monitoring.

The electronics and pneumatic system, together with an embedded battery (25.2 V nominal voltage and 4 Ah capacity), are contained in the backpack which has a total weight of $4 \mathrm{Kg}$.

\subsubsection{Control Strategy}

Actuation of the XoSoft QPAs is linked with the motion of the user. Due to the geometry of the exosuit the torque delivered by the actuation system is dependent on the moment arm $R_{k}\left(\alpha_{k}\right)$ subtended by the cord $L_{k}$, and by the cord elongation $\Delta L_{k}$, as described in section 3. This is shown in Figure 8 where the cord corresponds to the actuation line, which is the series connection of the TBC and EB from the proximal to the distal body attachments. The system stores and delivers energy as a consequence of the joint rotation. During the first phase of energy storage, a resistive torque is generated, which the user has to fight against. Once the joint starts rotating in the opposite direction (phase two), the system releases this accumulated mechanical energy. During the second phase, the system provides assistance to the user until the leg segment returns to the initial position (it corresponds to joint angle when the actuation has been engaged at the beginning of phase one). Therefore, it is possible to store and deliver different amounts of energy by modulating the timing of clutch engagement. The main control algorithm provides the segmentation of the gait cycle, which is shown in Figures 5, 6 as a logic state of a Finite State Machine (FSM). In correspondence of each state, the clutches can be set or reset.

The power delivered by the EB is controlled by detecting the phase in the gait cycle and using this information to engage and disengage the clutches. Figure 5 shows the flow chart of the state machine used to regulate these activations. The system tracks the gait phases sequence, using the foot contact data and knee angle, to determine when to engage, disengage or keep the clutch current state. The inputs to the stateflow are the foot contact signal and knee angle. The outputs of the stateflow are the onoff clutch activation signals. A configuration file is used to set and save each subjects' profile of who uses and tests the exosuit. It reports and specifies the actuation configuration such as the leg (left/right/bilateral), the joint and movement to be assisted (i.e.,

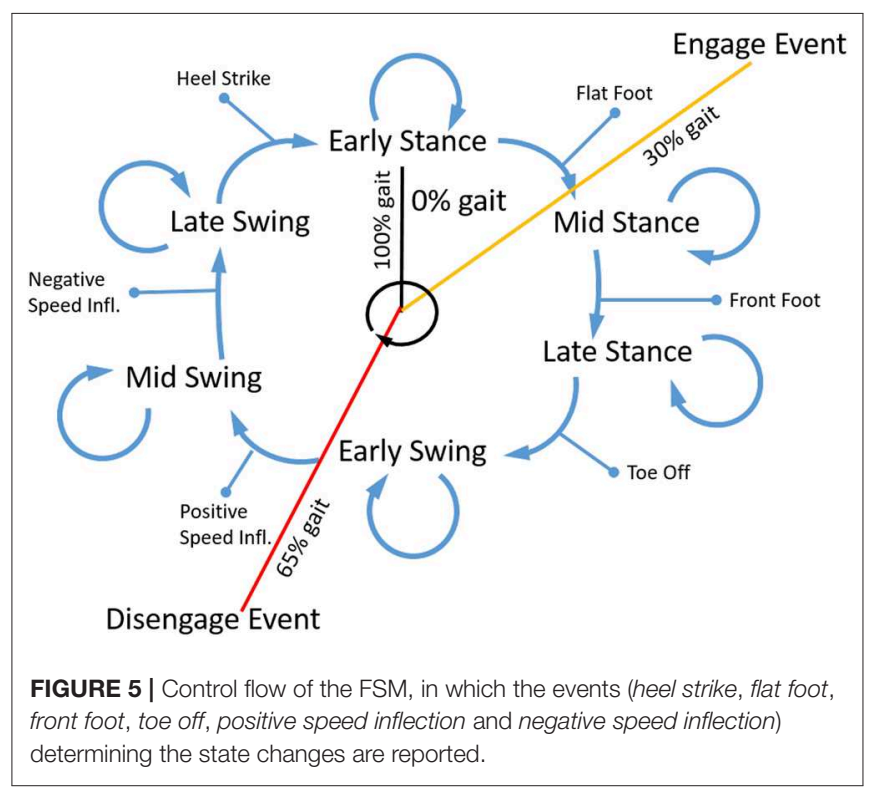




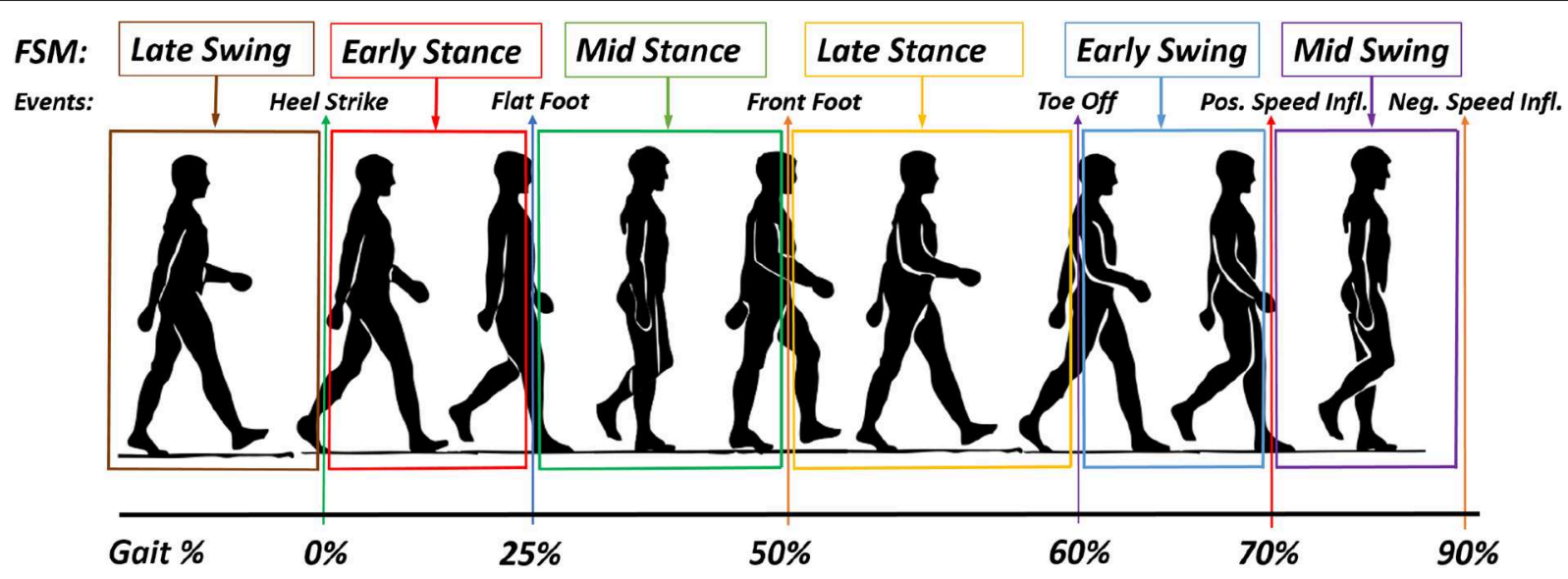

FIGURE 6 | Schematic of the gait event and FSM.

hip flexion and extension, knee flexion and extension, and ankle plantarflexion and dorsiflexion). The control of each clutch is set by means of the activation and deactivation instant during each percentage of gait. Therefore, the leg logic blocks detect the gait events sending the appropriate signals to the activation blocks, where the corresponding clutch control signals are triggered. Thus, the device is controlled with an open-loop algorithm. Apart from each of the clutch states, on the high level of the control, there is not a physical quantity that has to be controlled.

In the Beta 1 prototype, knee angle detection was unavailable. A simpler segmentation approach was used, and it relied on the time measurements between consecutive toe off (TO) and heel strike (HS). Thus, the swing phase estimation was based on the timing of the previous step. This simple approach is limited to scenarios where smooth changes in cadence are assumed. Therefore, to improve the gait segmentation, this method based on knee angle detection has been developed.

Based on the data from the shoe insole sensors and the IMUs, the controller is able to determine the six gait phases that form the finite state machine (FSM) (early stance, mid stance, late stance, early swing, mid swing, and late swing). These are shown in Figure 6. The assistance provided to the user, which is based on the user needs, is designed accordingly to the actuation configuration and the actuation strategy (clutch engagement and disengagement events expressed as gait percentage). The control designer, (essentially the therapist) may select the point, expressed as a percentage, of gait when the actuation should engage and disengage. The control system then processes this information to determine the correct timing to switch the actuator state. For every subsequent gait cycle, each duration os each segment is regenerated based on the previous gait's total time.

Figure 7 gives an example of assistance, separating the storing and releasing phases, for each assisted joint. Because of the design of the $Q P A$, the actuator needs to store energy to be able to provide assistance. In Figure $7 \mathbf{A}$, an example of hip flexion actuation is presented. The first vertical line represents the instant of TBC engagement (at about 15\% of the gait cycle). Naturally, the storing phase ends with the minimum angle reached by the joint (at about 50\%), then the releasing phase starts. The releasing phase will terminate as soon as the initial joint angle (instant of engagement) has been reached or as soon as the $T B C$ is disengaged by the controller. In this example, the releasing phase ends at approx. the $75 \%$ of the gait cycle. Figures 7B,E represent the knee movement (flexion and extension respectively) and show the ranges over which energy is stored and released. Since the knee angle rotation plot has two local maximums, two storing phases $\left(S_{1}\right.$ and $\left.S_{2}\right)$ and releasing phases $\left(R_{1}\right.$ and $\left.R_{2}\right)$ are possible. Of course, this will result in a different sensation for the user, and different assistance profiles (Di Natali et al., 2019 assesses the knee flexion control strategy). Figure $7 \mathrm{~F}$ shows the two possible actuation strategies for ankle dorsiflexion. Thanks to the two local maximums, the ankle dorsiflexion can be assisted during the whole gait cycle $\left(R_{1}\right.$ and $\left.S_{1}\right)$ or $\left(R_{2}\right.$ and $\left.S_{2}\right)$ around the toe-off event (60\% of gait cycle). The control strategy $S_{2}-R_{2}$ can be used to reduce the foot drop issue.

\section{MODELING}

A wearable exosuit/exoskeleton is designed to aid the user by applying a percentage of the needed power to accomplish the task. In this instance, the exoskeleton is applying force on the user's leg segments. Each $Q P A$ provides pulling forces between the proximal and distal body attachments. The body attachments are below and above the assisted joint, as shown in Figure 8. The force generated by the EB is a function of its elongation. The interaction with the user is important since joint motion will drive the EB by providing elongation. Figure 8 summarizes the main mathematical elements related to the force/torque transmission. The cord length (the in-line combination of the TBC and EB) $L_{k}\left(\alpha_{k}\right)$, is a function of the joint angle $\alpha_{k}$, where the index $k$ could be the front or rear hip, knee and ankle joint. The user's joint rotation generates an elongation defined as $\Delta L_{k}$ 

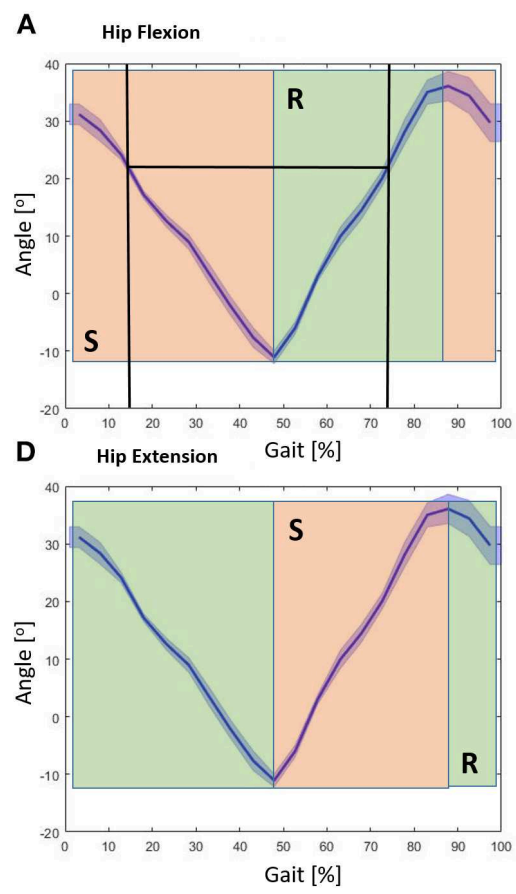
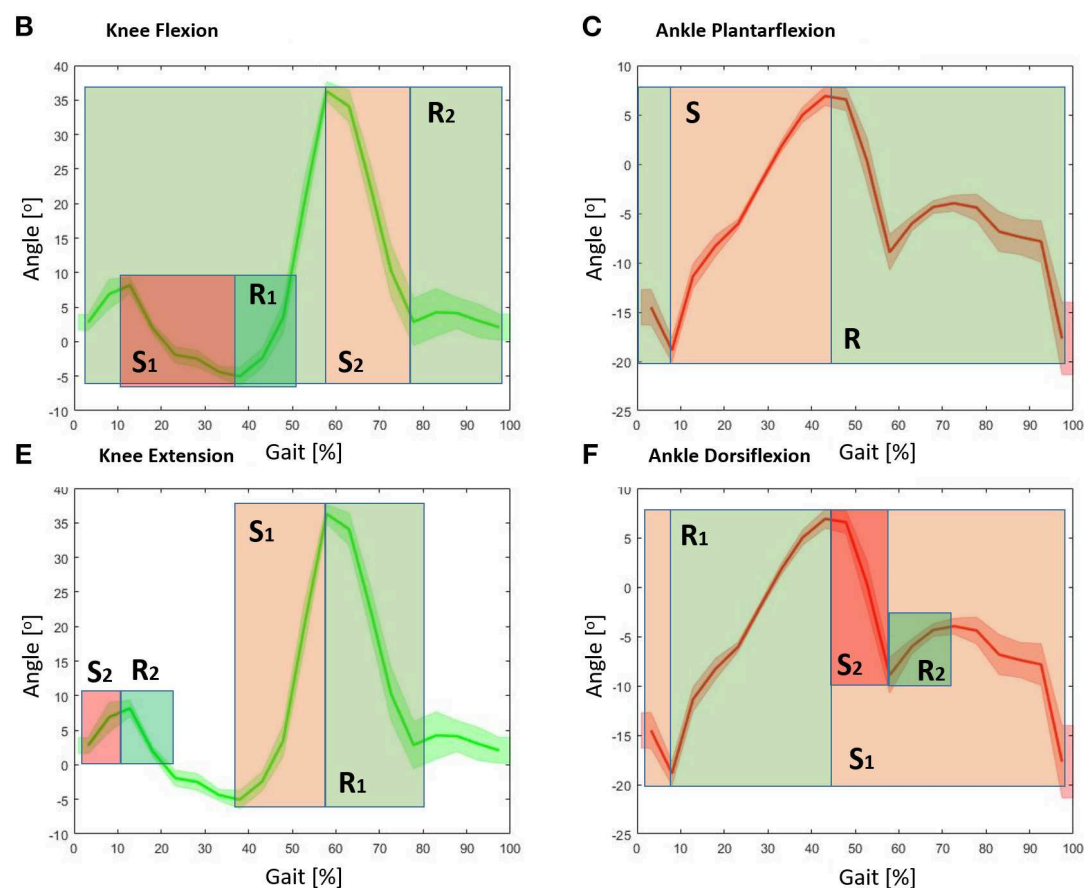

F Ankle Dorsiflexion Gait [\%]

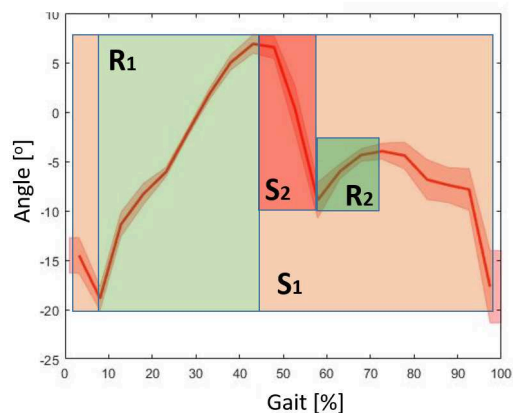

FIGURE 7 | Schematic of possible control strategies for each actuator group (hip, knee, and ankle) as a function of the joint angle. The green areas represent the energy releasing phase $(\mathrm{R})$, whereas the red areas represent the storing phase $(\mathrm{S})$ for each actuator grouping. (A) Representation of the hip flexion, the joint motion/rotation is associated with where energy could be stored and released. The black vertical lines show the instant of engagement and disengagement of the TBC, where the clutch is engaged at the $15 \%$ point of the gait cycle, and maximum releasing phase at $75 \%$. (B) Storing and releasing phases associated with the knee flexion. (C) Storing and releasing phases associated with the ankle plantarflexion. (D) Storing and releasing phases associated with the hip extension. (E) Storing and releasing ranges associated with the knee extension. (F) Storing and releasing phases associated with the ankle dorsiflexion.

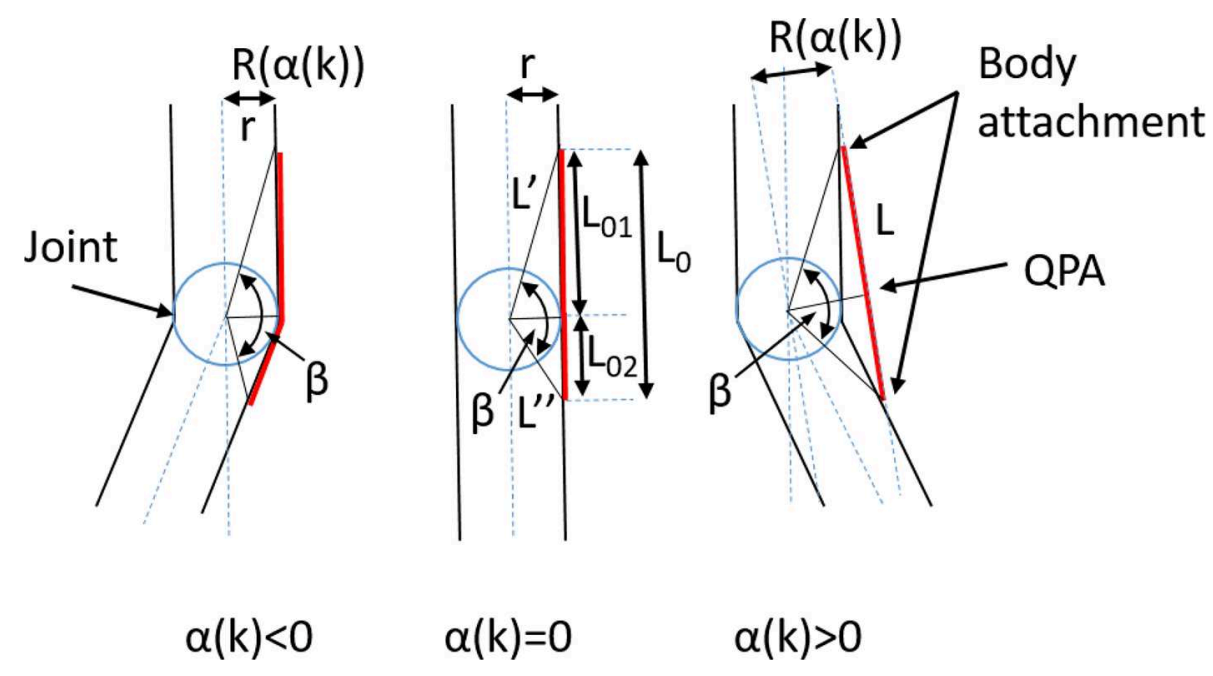

FIGURE 8 | Geometrical representation of the actuation unit.

on the cord. The total cord length is governed by Equation (2).

$$
L_{k}\left(\alpha_{k}\right)= \begin{cases}\sqrt{L_{k}^{\prime 2}+L_{k}^{\prime \prime 2}-2 L_{k}^{\prime} L_{k}^{\prime \prime} \cos \left(\beta_{k}\right)} & \text { if } \alpha_{k} \text { is } \geq 0 \\ L_{0 k}+r_{k} \alpha_{k} & \text { if } \alpha_{k} \text { is }<0\end{cases}
$$

where

$$
\begin{aligned}
& L_{k}^{\prime}=\sqrt{L_{01 k}^{2}+r_{k}^{2}} \\
& L_{k}^{\prime \prime}=\sqrt{L_{02 k}^{2}+r_{k}^{2}}
\end{aligned}
$$


The angle $\beta_{k}$ is equal to:

$$
\beta_{k}=\pi-\alpha_{k}-\arctan \left(\frac{r_{k}}{L_{01 k}}\right)-\arctan \left(\frac{r_{k}}{L_{02 k}}\right)
$$

$L_{0 k}$ is the length of the cord formed by the TBC and EB assembly in its neutral position. This occurs when $\alpha_{k}=0$, it is equal to:

$$
L_{0 k}=L_{k}^{\prime}+L_{k}^{\prime \prime}=L_{01 k}+L_{02 k}
$$

The torque provided by the actuation system is strongly influenced by the moment arm $R_{k}\left(\theta_{k}\right)$ subtended by the cord $L_{k}$, and by the cord elongation $\Delta L_{k}$. The cord elongation is completely provided by the EB's elongation, whereas the $R_{k}\left(\alpha_{k}\right)$, function of the joint angle is expressed as:

$$
R_{k}\left(\alpha_{k}\right)= \begin{cases}r_{k} & \text { if } \alpha_{k} \text { is }<0 \\ \frac{L_{k}^{\prime} L_{k}^{\prime \prime}}{L_{k}\left(\alpha_{k}\right)} \sin \left(\beta_{k}\right) & \text { if } \alpha_{k} \text { is } \geq 0\end{cases}
$$

As a first approximation, $R_{k}\left(\alpha_{k}\right)$ is considered to be always aligned to the sagittal plane. Therefore, the torque generated on the assisted joint is proportional to the EB's force, $f_{k}$ as shown in Equation (8).

$$
\tau_{k}=R_{k}\left(\alpha_{k}\right) f_{k}=R_{k}\left(\alpha_{k}\right) K_{E B}\left(\Delta L_{k}^{E B}\right)
$$

Where $K_{E B}\left(\Delta L_{k}^{E B}\right)$ is the third order EB's stiffness function reported in Table 4 and $\Delta L_{k}^{E B}$ is the amount of elongation generated by the EB.

Tasks conducted by the test subject while wearing the soft exoskeleton are characterized by a particular amount of torque $\tau_{t}$ at each joint. The exoskeleton aids the user by providing the torque $\tau_{e x}$, as defined in Equation (8). The mechanical system, comprising both the user and exoskeleton, requires an amount of energy for the storing phase. This energy is provided by the user during a specific phase of the gait cycle. The total absorbed energy, into the $\mathrm{EB}$, is then returned by the exoskeleton in the form of assistance.

To analyze the system's behavior, the power associated with the task, which is the combination of the torque and angular velocity of each joint, is calculated in Equation (9).

$$
P_{t}=\tau_{t} \dot{\alpha}_{t}
$$

It is then possible to calculate the assistance provided by the exoskeleton by computing the ratio of powers $(\Lambda)$ as:

$$
\Lambda=\left|\frac{P_{t}-P_{e x}}{P_{t}}-1\right|
$$

where $P_{t}$ is the measured power of the user when not wearing the exoskeleton, and $P_{e x}$ is the power generated by the exoskeleton. $\Lambda=0$ means that the exosuit is not providing any assistance, while $\Lambda=1$ represents $100 \%$ of assistance (the exoskeleton assistive power is instantaneously equal to the user power).

\section{EXPERIMENTAL EVALUATION}

This section characterizes the $T B C$ and pneumatic system. This is followed by an assessment on a healthy subject (the subject had a traumatic event in 2012 on its right ankle, causing compensatory movements) to evaluate, from a control system perspective, the assistance provided (Figure 9). The test evaluation is compliant with the experimental protocol accepted by the Ethical Committee of Liguria, Italy (protocol reference number: CER Liguria 001/2019) and the subject gave written informed consent in accordance with international guidelines. The software was evaluated during a 10 min walking task on a treadmill, during which the exoskeleton's parameters, together with motion tracking, were recorded. A second test was conducted along a $10 \mathrm{~m}$ straight path where the kinematics of the limbs and ground reaction forces were measured to analyze the effectiveness of the transmitted assistance.

\subsection{Actuator Characterization}

The actuators have been evaluated mechanically, pneumatically and electrically to characterize the actuation timing, the generation of forces and the power consumption. Each actuator is composed of an $E B$ and the TBC. Each $T B C$ is supplied by a pneumatic system, which is controlled by electromagnetic valves. The $E B$ characterization has previously been evaluated in Di Natali et al. (2019). As previously mentioned, the stiffness characteristic of these latex EBs is not linear. A 3rd order polynomial has been used to fit the elastic behavior as a function of the percentage elongation $\left(\Delta L_{k}\right)$, as in Equation 1. The TBC blocking force has been evaluated by measuring the resistive force generated as the vacuum pressure is increased. An Instron (ITW, Glenview, IL) force measurement system was used to characterize the TBC (Figure 10). The tests were repeated ten times for the lower leg clutch (the smaller clutch of the $T B C$ family, Table 2) with $100 \%$ overlap between the two layers of the device. The tests were performed starting with a null vacuum pressure $(101 \mathrm{kPa})$ and reducing (increasing vacuum) in steps of $10 \mathrm{kPa}$, to $50 \mathrm{kPa}$. The profile although not linear, shows a smooth repeatable response increasing to a maximum blocking force of $400 \mathrm{~N}$ at a pressure of $50 \mathrm{kPa}$. A maximum blocking force of $540 \mathrm{~N}$ was recorded at a pressure of about $42 \mathrm{kPa}$, however at this point the TBC broke. Therefore for safe operation a peak pressure of $50 \mathrm{kPa}$ was selected, which gave a peak blocking force of $400 \mathrm{~N}$. In absence of vacuum, the average recall force is $7.5 \mathrm{~N}$. This is due the intrinsic recall elastic element into the $T B C$.

As each actuator forms a load for the vacuum system it is believed that increasing the number of actuators could impact the operational timings of the exoskeleton. To test this the activations timing for single actuator and four actuators were tested. In these test the single actuation and the four actuator units were connected to the vacuum system. To determine the time needed to actuate these clutches, ten tests were performed for each of two families of clutches. The times to create a vacuum pressure of $70 \mathrm{kPa}$ were measured. The time to actuate the upper leg clutches, is $0.10 \pm 0.006 s$ for the single clutch and $0.18 \pm 0.026 s$, if four clutches are connected to the vacuum generator. For the lower leg clutches, the time of actuation is $0.08 \pm 0.018 s$ for the 


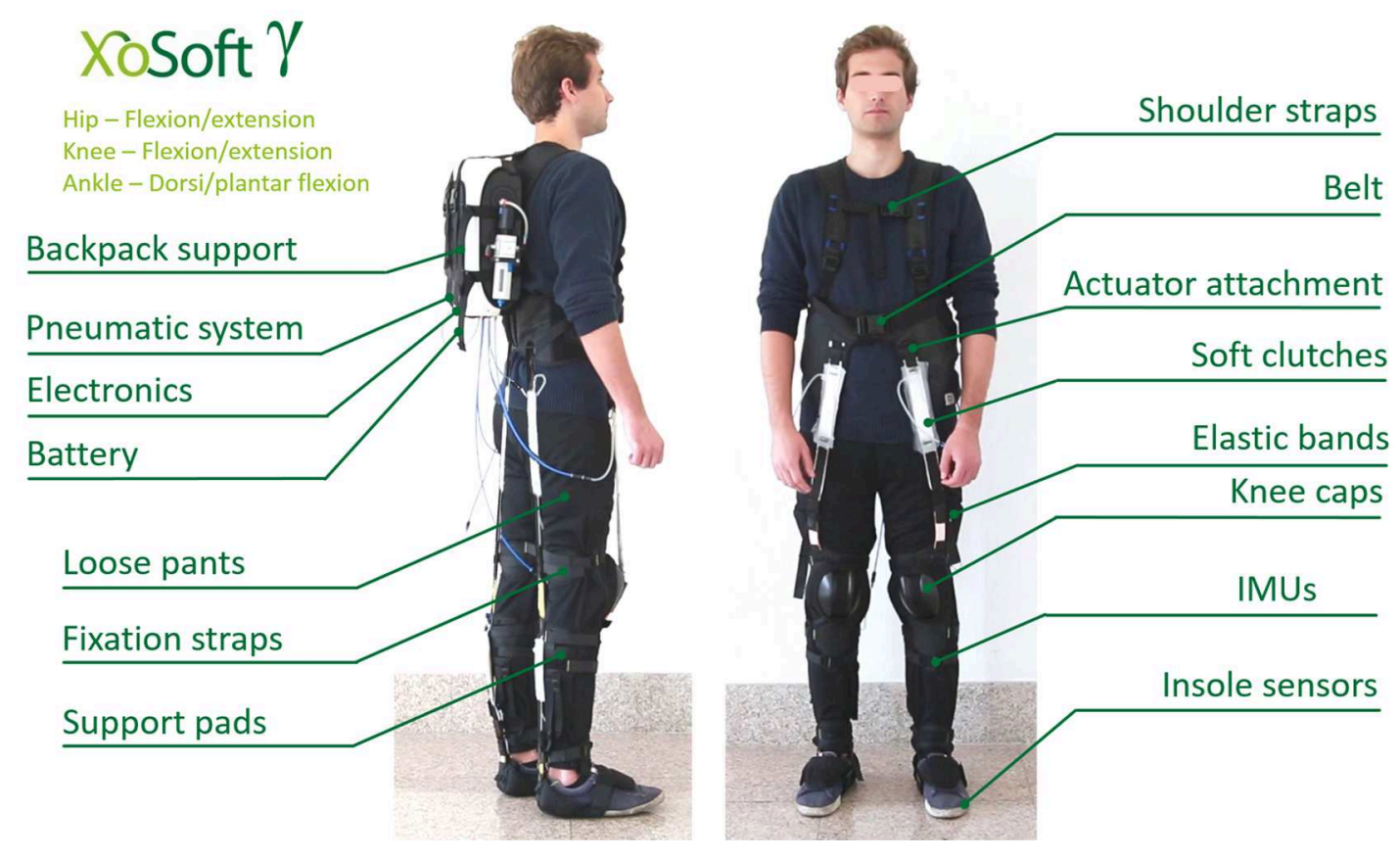

FIGURE 9 | The system overview of the Gamma prototype used during the experimental evaluation. Written informed consent was obtained from the individual pictured in figure.

\section{Blocking Force $(\mathrm{N})$}

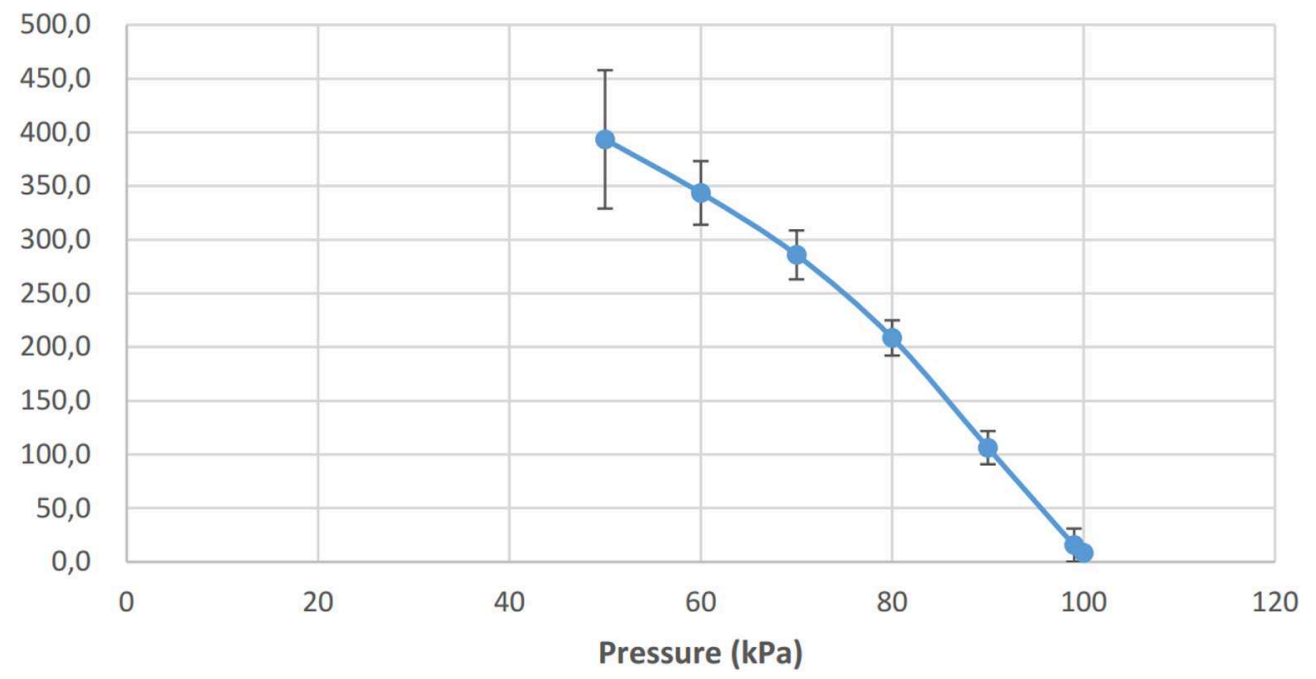

FIGURE 10 | The average maximum force of TBC recorded at different applied pressures (for easy conversion 1atm $=101.3 \mathrm{kPa}, 50 \mathrm{kPa}$ corresponds to $50 \%$ of vacuum).

single clutch, and $0.12 \pm 0.010 \mathrm{~s}$, if four clutches are connected to the vacuum generator. The releasing phase was also measured. The time, always constant, was $0.1 s$ no matter of how many clutches were controlled at the same time. Therefore, we could assume an average time to energize and released each actuation of $0.12 \mathrm{~s}$. The test shows a dependency between the time of actuation and the number of clutches connected to the same vacuum generator. This is probably because the characteristics of the Venturi method used to generate vacuum and the selected pneumatic circuitry (shown in Figure 4). As previously mention 
in section 2.1.3, the Venturi system employs high air flow to suck air generating vacuum. Thus, the characteristic latency measured to energize the clutches is due to the air contained into the clutch and along the pneumatic lines of the exoskeleton.

To evaluate exoskeleton autonomy, the Gamma prototype was tested on a treadmill for approximately $10 \mathrm{~min}$ at a constant speed of $3 \mathrm{~km} / \mathrm{h}$. To produce comparable results with the system assessed in the section 4.3 the device was configured with six actuators (two actuators for hip flexion, two actuators for knee flexion, and two for ankle plantarflexion). During the walking task, the actuator engagement sequence, accordingly to the designed control strategy (more details are in section 4.3), was driving the actuation of each clutch at a frequency of 0.85 $\mathrm{Hz}$. During testing, the overall current consumption (including $\mathrm{CPU}$, sensors, communication and actuations) was monitored and measured. The power consumption, in terms of average current, is $0.17 \mathrm{~A}$ for each actuator. Regarding autonomy, the Gamma prototype equipped with six actuators assisting walking at $3 \mathrm{~km} / \mathrm{h}$ last approximately $4 \mathrm{~h}(235.3 \mathrm{~min})$.

To assess the efficacy of the exoskeleton, the forces generated by the EBs during operation should be measured. A possible method would consist on measuring the EB's elongation with optical methods. In Di Natali et al. (2019) such data was gathered during the system assessment of the Betal prototype. During the tests, different control strategies were evaluated and the EB elongations measured using optical system in combination

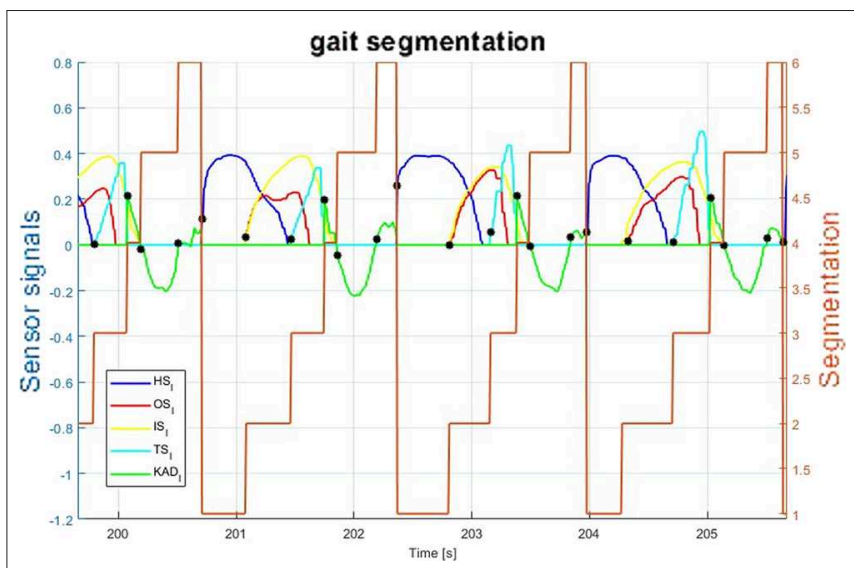

FIGURE 12 | Segmentation based on sensors fusion. The signals are as follows: (HS) heel pressure signal, (OS) outside insole pressure signal, (IS) inside insole pressure signal, (TS) toe pressure signal and (KAD) the derivative of the knee angle.

with two in-built markers. Two optical markers were installed at both ends of each EB to directly measure their elongation during operation. Since the optical system was not available for the current system evaluation, the geometrical model presented in section 3 is validated on the data gathered during the
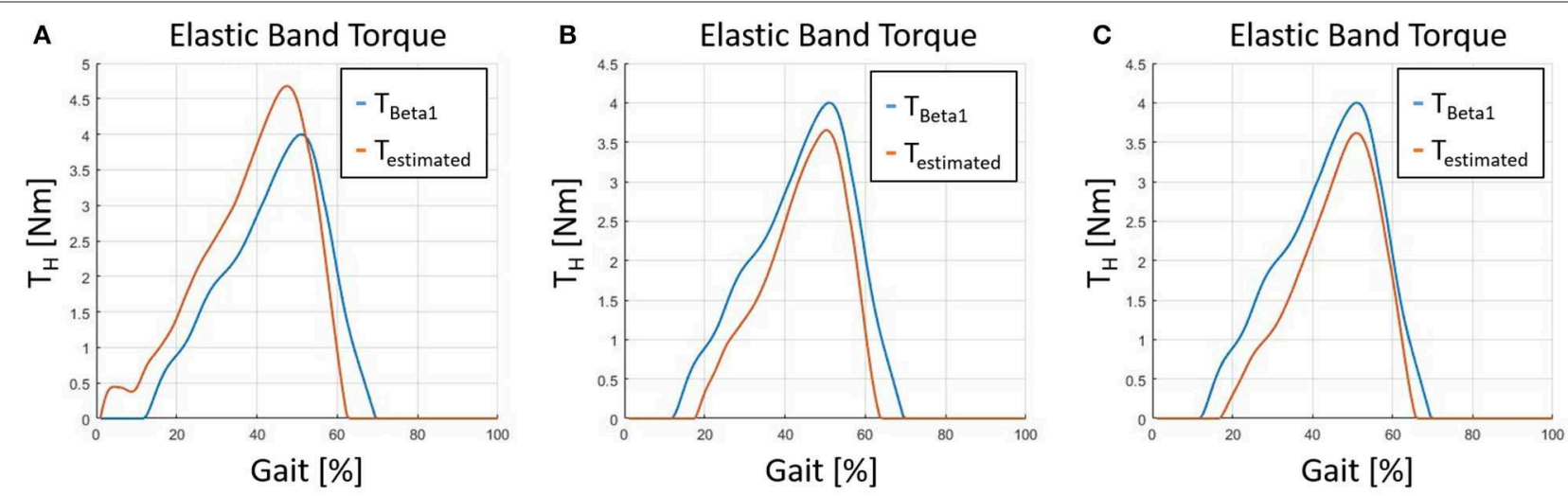

D Elastic Band Torque
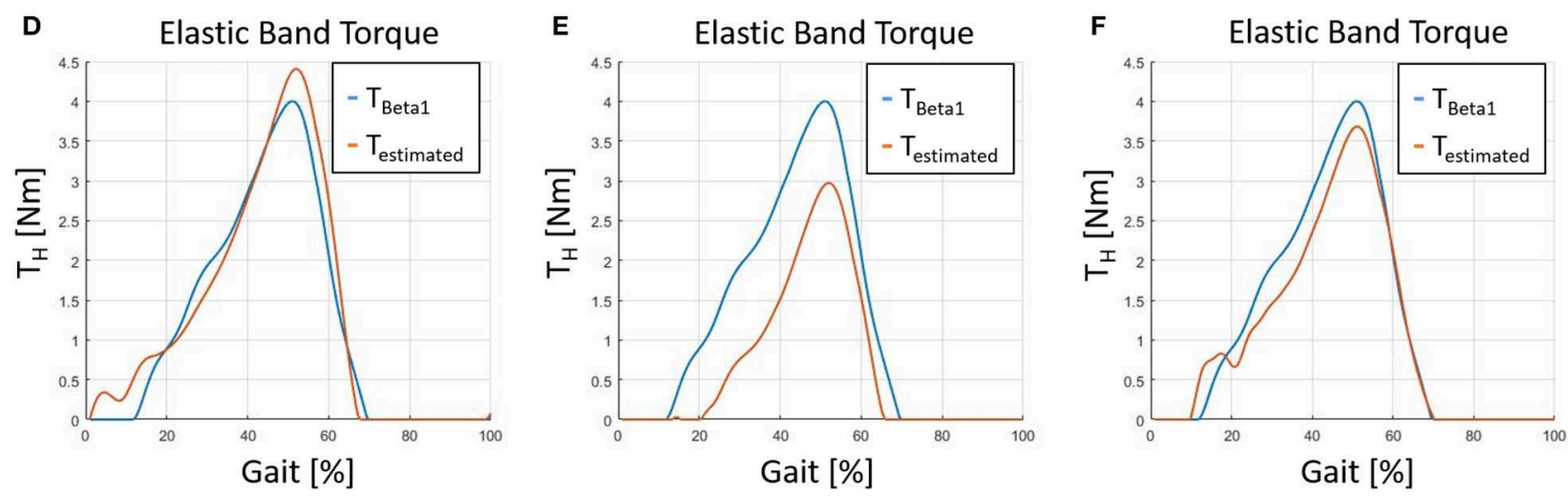

FIGURE 11 | The figure shows six different tests where the torque was measured during the assessment of the Beta1 prototype in Di Natali et al. (2019) against the torque estimated with the geometrical model presented in section 3. (A) Test 1, (B) test 2, (C) test 3, (D) test 4, (E) test 5, and (F) test 6. 
Beta1 prototype assessment presented in Di Natali et al. (2019) (employing the measurements of the EB's elongation). The torque generated by the EB elongations were estimated with the model presented in section 3. Figure 11 reports the results of the measured torque of the Beta 1 prototype against the estimated torque employing the geometrical model. The mean absolute error and standard deviation are $0.25 \pm 0.31 \mathrm{Nm}$, whereas the mean relative error with respect to direct measurement and its standard deviation are $6.52 \pm 8.13 \%$. Thanks to the validation of the geometrical model, and considering an error below 7\%, the model can be considered as having a good level of reliability. The geometrical model is used to estimate the torque generated by the exosuit for the calculation of its performance in section 4.3.

\subsection{Control Algorithm Characterization}

The control algorithm was assessed by walking on a treadmill for $10 \mathrm{~min}$ at a constant speed of $3 \mathrm{~km} / \mathrm{h}$. Sensor readings of the exoskeleton were recorded. To record full body kinematic and ground contact data, motion tracking was carried out using a Xsens wearable motion tracking system (MTw Awinda 3D Wireless Motion Tracker, Xsens Technologies B.V. Enschede, The Netherlands). As previously mentioned, the control algorithm is based on sensor fusion of data from two insole pressure sensors and four IMUs on the thighs and shins. This set-up identifies, for the right and left sides, six events: (i) heel strike, (ii) flat foot, (iii) front foot, (iv) toe off, (v) maximum positive speed, and (vi) minimum negative speed of the knee during swing. Figure 12 shows the segmentation achieved by applying this sensor fusion based algorithm. Thus, the gait is divided in six segments, three during stance and three during swing as follows: early stance (ESt), mid stance (MSt), late stance (LSt), early swing (ESw), mid swing (MSw), and late swing (LSw). For each segment the mean duration and standard deviation are reported in Tables 5, 6 for right and left side, respectively. The tables show repetitive and reproducible values of time lengths of each segment with a mean standard deviation over mean segment of about $19 \%$. Each of the six segments were determined, for each gait cycle during the $10 \mathrm{~min}$ test, without generating any segmentation error, thus, corresponding to a $100 \%$ of execution. These events (gait percentage) are used to identify the consecutive instants of the segmentation algorithm. Differences in segment duration between the right and left side (see Tables 5, 6) are due to walking pattern asymmetry. The user reported a traumatic event on the right ankle that caused such pattern asymmetry. In fact, the asymmetric gait pattern has been properly detected by the actual segmentation together with the suggested wearable sensors, confirming the validity of the proposed method. From a control point of view, these events represent the gait phases in which the QPA may store or release energy as demonstrated in Figure 7. Also, it is important to underline that the storing phase may occur only if the $E B$ is elongated. Thus, by considering minimum and maximum angular displacement related to the particular joint motion, the control may be set accordingly to trigger the $Q P A$ within the desired gait areas. The next section details a particular control scenario to evaluate the assistance of the QPA on the hip, knee and ankle.
TABLE 5 | Right side segmentation timing and gait percentage.

\begin{tabular}{lcccc}
\hline Segment & $\begin{array}{c}\text { Mean } \\
\text { segment } \\
\text { duration [s] }\end{array}$ & $\begin{array}{c}\text { STD } \\
\text { segment } \\
\text { duration [s] }\end{array}$ & $\begin{array}{c}\text { Mean duration } \\
\text { percentage [\%] }\end{array}$ & $\begin{array}{c}\text { Gait } \\
\text { percentage } \\
\text { [\%] }\end{array}$ \\
\hline ESt & 0.4279 & 0.1225 & 27.47 & 27.47 \\
MSt & 0.3647 & 0.1036 & 23.42 & 50.89 \\
LSt & 0.0855 & 0.0516 & 5.49 & 56.38 \\
ESW & 0.1961 & 0.0437 & 12.59 & 68.97 \\
MSW & 0.3413 & 0.0396 & 21.91 & 90.88 \\
LSW & 0.1420 & 0.0790 & 9.12 & 100.00 \\
\hline
\end{tabular}

TABLE 6 | Left side segmentation timing and gait percentage.

\begin{tabular}{lcccc}
\hline Segment & $\begin{array}{c}\text { Mean } \\
\text { segment } \\
\text { duration [s] }\end{array}$ & $\begin{array}{c}\text { STD } \\
\text { segment } \\
\text { duration [s] }\end{array}$ & $\begin{array}{c}\text { Mean duration } \\
\text { percentage [\%] }\end{array}$ & $\begin{array}{c}\text { Gait } \\
\text { percentage } \\
\text { [\%] }\end{array}$ \\
\hline ESt & 0.3336 & 0.1158 & 21.50 & 21.50 \\
MSt & 0.3868 & 0.1127 & 24.93 & 46.43 \\
LSt & 0.2185 & 0.0891 & 14.08 & 60.51 \\
ESw & 0.1187 & 0.0365 & 7.65 & 68.16 \\
MSW & 0.3296 & 0.0348 & 21.25 & 89.41 \\
LSW & 0.1643 & 0.0503 & 10.59 & 100.00
\end{tabular}
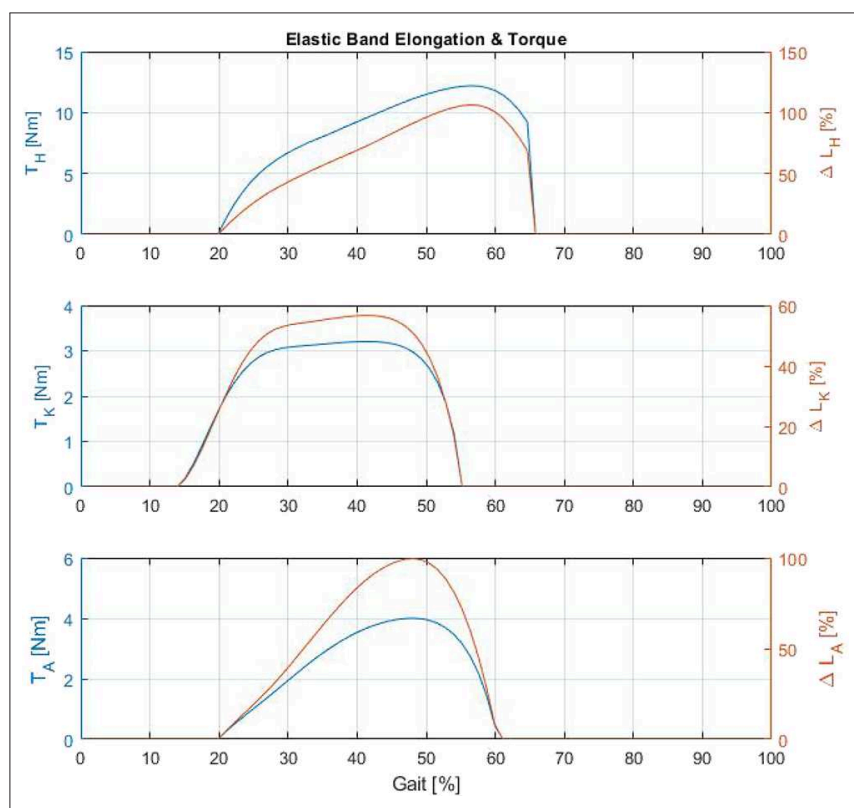

FIGURE 13 | Simulation of elastic band torque and elongation for hip, knee, and ankle.

\subsection{Subject Assessment}

In this section, the device assessment is addressed. The device was tested on a $10 \mathrm{~m}$ long platform with two force plates (BTS P6000, BTS SpA bioengineering, Italy) located about three quarters of way along the course. The tests were completed ten times, during which ground reaction forces on both sides of the body and full body kinematics were measured. Motion 
capture was recorded using the Xsens system. The AnyBody modeling system (AnyBody Technology A/S, Niels Jernes Vej 10, DK-9220 Aalborg Ø, Denmark) was used to extract and calculate the joint torques from measurement of kinematics and the ground reaction forces. The exoskeleton was configured with six assistive actuators at the hip, knee, and knees on both sides of the body. Three different control sequences were used for frontal hip assistance (hip flexion), rear knee assistance (knee flexion) and rear ankle assistance (ankle plantarflexion). In particular the QPAs assisting the hip flexion were triggered from 20 to $65 \%$ of the gait cycle. Figure 7 shows that the energy releasing phase is effective until at least $75 \%$ of gait cycle. By disengaging the $T B C$ at the $65 \%$ point, although there is still elastic energy in the system, an instant drop in the assistive torque and elongation occurs (Figure 13). This behavior shows the quality of a QPA. It is able to modulate the energy transmitted to the user, by selecting both the instant of activation and release of the actuation during operation. The second pair of QPAs assist flexion of right and left knees, with the control triggered at $15 \%$ of the gait cycle and clutch disengaged at 55\%. Finally, the last pairs of QPAs for ankle assistance are triggered between 20 and $65 \%$ of gait. Since the ankle angle can exceed the value assumed at the trigger event, the torque transmitted decays to a null value before the end of the control sequence. To demonstrate this, the measured values of ankle shown in Figure 14C (blue trend) shows that the ankle assumes the same angular values at the 20 and $60 \%$ of gait cycle. This is also confirmed by the geometrical model, shown in Figure 13, where the $E B$ elongation and torque transmission over the ankle joint reach a null value at $60 \%$ of the gait cycle. The $E B$ elongation and torque generation are based on the model presented in section 3 and validated in section 4. Figure 14 shows the angular displacements, torque and power at the hip, knee and ankle when wearing the Gamma prototype and when not wearing it $(\mathrm{NoXoS})$, respectively. The torque and power for the $E B$ are displayed, showing the net effect of the exoskeleton in assistance mode. When the power is negative the elongation of the $E B$
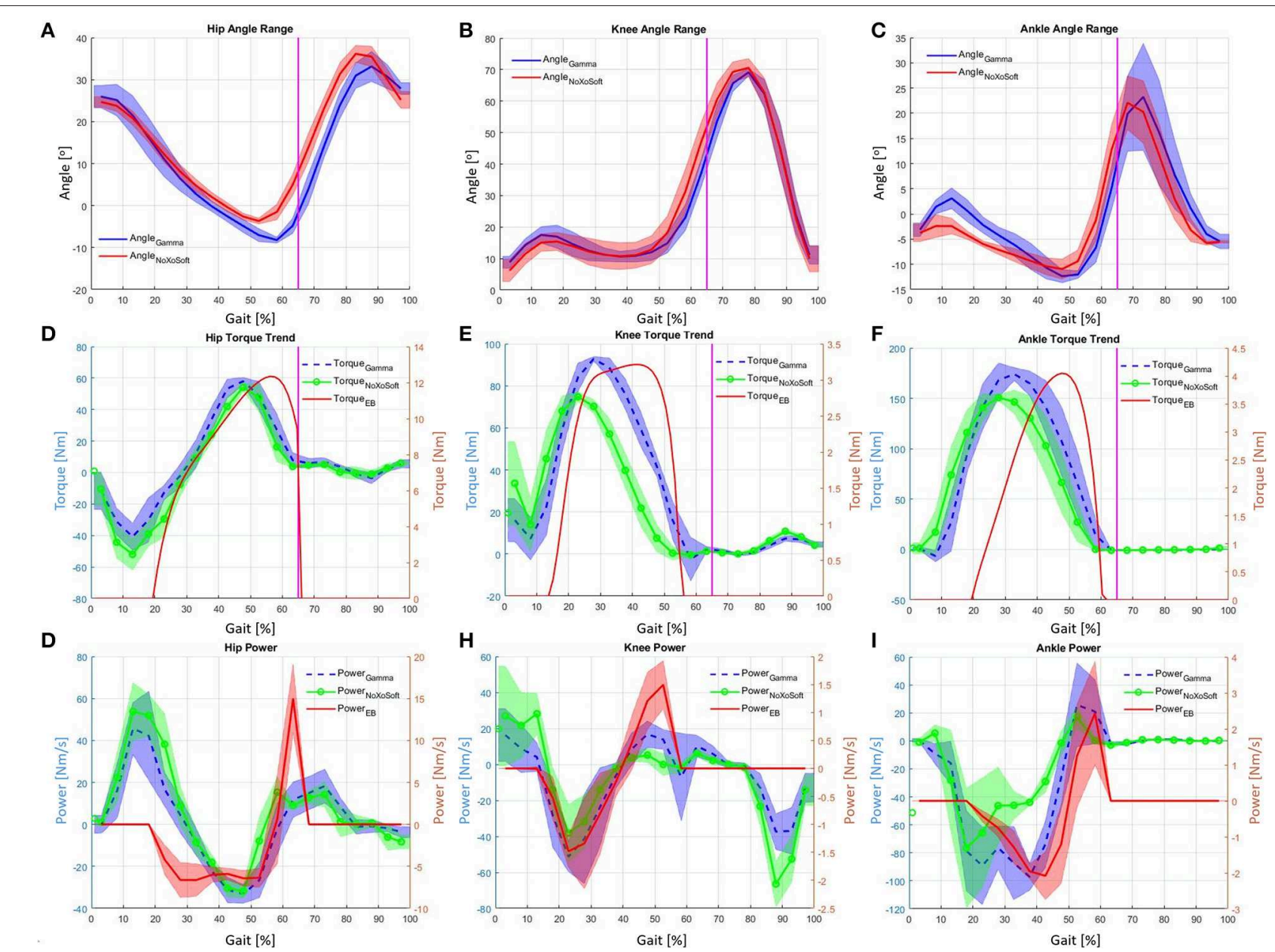

FIGURE 14 | (A) Hip angle range wearing XoSoft and not wearing XoSoft. (B) Knee angle range wearing XoSoft and not wearing XoSoft. (C) Ankle angle range wearing XoSoft and not wearing XoSoft. (D) Hip torque wearing XoSoft, not wearing XoSoft and with the EB alone. (E) Knee torque wearing XoSoft, not wearing XoSoft and with the EB alone. (F) Ankle torque wearing XoSoft, not wearing XoSoft and with the EB alone. (G) Hip power wearing XoSoft, not wearing XoSoft and with the EB alone. (H) Knee power wearing XoSoft, not wearing XoSoft and with the EB alone. (I) Ankle power wearing XoSoft, not wearing XoSoft and with the EB alone. 
extracts energy from the system (exoskeleton-human) by storing energy, then the net assistance occurs only when the power is positive. The assistive torque causes variations on the measured torque plots. These changes appear as increments or decrements in the total joint torque as the system needs to overcome the $E B$ resistance or receives its net assistance.

Figure 15 shows the estimate of the exosuit's assistance applying the Equation (10). Which is determined by the ratio of the difference of power at the specific joint with and without the exosuit, with respect to the mechanical power generated at the same joint without having the exoskeleton worn. The power generated wearing the exoskeleton is calculated coupling the measured joint speed and the joint torque estimated applying the geometrical model, which is validated in section 4.1. The diagram clearly shows that the assistance provided may be greater than $100 \%$, because the released net assistive power, in certain gait phases, exceeds the joint power. Thus, the effectiveness of the assistance becomes significant. The power extracted from the system during the $E B$ storage phase is less than the characteristic power of the particular gait section. Thus, the storage phase does not affect the overall $\Lambda$ index, and the user does not feel the extraction of energy from the system. The overall average assistive power measured in terms of the $\Lambda$ index (shown in Figure 15) for the hip is $26.6 \%$, for the knee $9.3 \%$ and for the ankle joint $12.6 \%$. The maximum value of assistive power for the hip is $113.6 \%$, for the knee $93.2 \%$ and for the ankle joint $150.8 \%$.

\subsection{Discussion and Exoskeletons Overview} In this section, we compare the XoSoft Gamma prototype's main features against the key research and commercial mobile exoskeletons cited in section 1 of this paper and following recent review papers (Li et al., 2015; Yan et al., 2015). Table 7 reports the characteristics of interest of the selected exoskeletons. The main differences is the amount of generated assistance, which

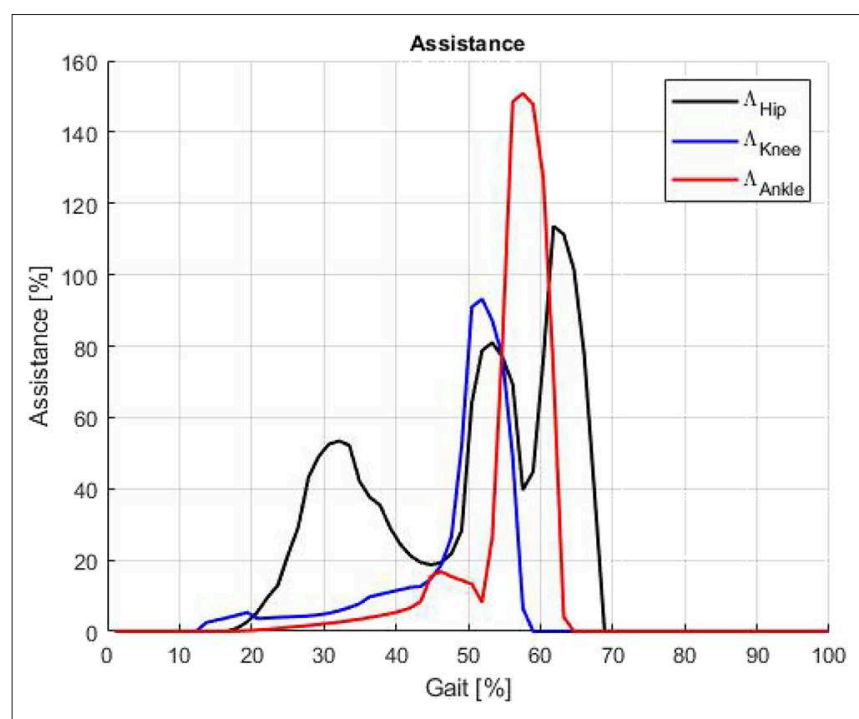

FIGURE 15 | Assistance on hip, knee and ankle expressed as $\Lambda$ function: the overall average power assistance normalized on the gait are $26.6 \%$ for hip, 9.3\% for knee, and $12.6 \%$ for ankle. also defines the user target. In fact the first two exoskeletons of Table 7, aim to restore the completely loss of mobility for paraplegic patients. Thus, these exoskeletons targets to regain locomotion ability by generating the total amount of joint torque ( $100 \%$ of torque ratio). A second device family aims to provide a partial power to give an aid during walking to people suffering from muscular weakness. An example are elderly people, who need a device able to generate a partial amount of the joint torque. Therefore, if the exoskeleton does not restore total walking faculty the requested assistance reduces within a third of the total joint torque. The natural trend, visible in research, is to move toward a simplification of the structure, which does not necessarily need to be hard but it may become soft. As well, the actuation may be simplified moving from an active to a quasi-passive assistance as demonstrated in the presented device (XoSoft Gamma). The main differences between a hard structure and a soft structure in terms of comfort and encumbrance are well-underlined in section 1. In general, hard structure are recommended if large amount of torque has to be generated to specific joints. But if that is not the case, the inconveniences related to a heavy and uncomfortable structure arise. Moving from hard to soft a drop of torque ratio is evident, in fact no more than a quarter of the total torque can be generated to the exoskeleton employing soft structure. On the contrary assistive exoskeletons such as the EXPOS and the WWH, can generate a third or half of the total joint torque respectively. Focusing on soft exoskeletons, it is evident that the most common actuation of the selected exoskeletons is the active one. There is only one QPA which employing passive elements (e.g., elastic bands) which is able to generate a comparable torque ratio with respect to the active systems but using simpler actuation system.

\section{CONCLUSIONS}

This paper presents a novel design and experimental validation of a modular soft exoskeleton for lower limb assistance. Each singular component of the system, such as actuation module, control, and software are evaluated in terms of power consumption, reaction time and force generation. The overall exoskeleton performance is assessed with a healthy user during a walking task. Torque and mechanical power are evaluated, bilaterally, at the three assisted joints (e.g., hip, knee, and ankle). The measured performances in terms of overall assistance, averaged along the gait cycle, are $26.6 \%$ for the hip, $9.3 \%$ for the knee and $12.6 \%$ for the ankle joint. The maximum value of assistive power for the hip is $113.6 \%$, for the knee $93.2 \%$ and for the ankle joint $150.8 \%$.

The particular characteristic of this exosuit employing $Q P A$ to assist joints, as demonstrated in this study, is the ability to accumulate energy in a gait phase (e.g., stance) where the user is generating more power and then released when the joint torque is not very high (e.g., swing). Therefore, the exosuit drains energy from the user to elongate the EB and the user does not feel the extraction of energy from the system. Then, the system releases energy during a gait phase where both the user torque and the assistive torque are comparable. Thus, the released energy affects the user's energy balance more than the stored one. The result, indeed, shows that such system is able to produce a relative power 
TABLE 7 | Exoskeleton comparison.

\begin{tabular}{|c|c|c|c|c|c|c|c|c|}
\hline Exoskeleton name & $\begin{array}{l}\text { Struct. } \\
\text { typ. }\end{array}$ & Actuation & Joints & DOF & $\begin{array}{l}\text { Bilateral } \\
\text { actuation }\end{array}$ & $\begin{array}{l}\text { Bidirectional } \\
\text { actuation }\end{array}$ & Weight [Kg] & $\begin{array}{l}\text { Assistive } \\
\text { perform. } \\
\text { (Torque ratio) }\end{array}$ \\
\hline $\mathrm{HAL}^{+}$(Sankai, 2010) & Hard & AS (P) & $\begin{array}{l}\text { Hips, knees, } \\
\text { (ankles) }\end{array}$ & $4(2)$ & $\checkmark$ & $\checkmark$ & 15 & $100 \%$ \\
\hline $\begin{array}{l}\text { Vanderbilt lower-limb orthosis (Farris } \\
\text { et al., 2011) }\end{array}$ & Hard & AS & Hips, knees & 4 & $\checkmark$ & $\checkmark$ & 12 & $100 \%$ \\
\hline WWH (Nakamura et al., 2005) & Hard & AS & Knees & 2 & $\checkmark$ & $\checkmark$ & - & $50 \%$ \\
\hline EXPOS (Kong and Jeon, 2006) & Hard & AS & Hips, knees & 4 & $\checkmark$ & $\checkmark$ & $3^{* *}$ & $32 \%$ \\
\hline $\begin{array}{l}\text { Power assist wear (Sasaki et al., } \\
\text { 2013) }\end{array}$ & Soft & AS & Knees & 2 & - & - & 3.7 & Unknown \\
\hline Soft Exosuit (Awad et al., 2017) & Soft & AS & Hip & 1 & - & - & 3.2 & $12 \%$ \\
\hline $\begin{array}{l}\text { Soft wearable robotic suit (Jin et al., } \\
\text { 2017) }\end{array}$ & Soft & AS & Hips & 2 & - & - & 2.7 & $8 \%$ \\
\hline Myosuit (Schmidt et al., 2017) & Soft & AS & Hips, knees & 2 & - & - & 4.6 & $26 \%$ \\
\hline XoSoft Beta (Di Natali et al., 2019) & Soft & QPA & Hip, knee & 2 & - & - & 2.9 & $10 \%$ \\
\hline XoSoft Gamma & Soft & QPA & $\begin{array}{l}\text { Hips, knees, } \\
\text { ankles }\end{array}$ & 6 & $\checkmark$ & $\checkmark$ & 4 & $16 \%{ }^{*}$ \\
\hline
\end{tabular}

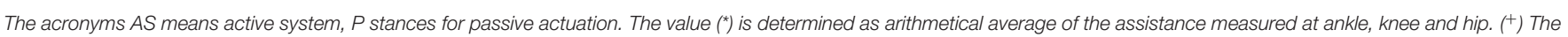

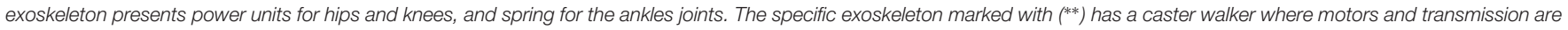
contained.

higher than $100 \%$ if compared to the actual instantaneous joint torque w/o the exoskeleton (it is shown in Figure 15 after the $50-55 \%$ of the gait cycle).

While a statistically relevant study was outside the scope of this current assessment study, these results are very promising and point toward positive findings overall. The device reveals a high potential for energy reduction as well as rehabilitation/motion assistance approaches to address small impairments on specific articulations as has been disclaimed in the following contributions (Graf et al., 2018; Poliero et al., 2018; Sposito et al., 2018; Di Natali et al., 2019). Further studies on patients are being conducted to validate the effectiveness of the exoskeleton. Further improvements to the exoskeleton will aim to develop proprioceptive solutions for sensory based actuators, but also specific soft sensing (Totaro et al., 2017) to measure user's angular joint and enable a more accurate control strategy. Future works will address how XoSoft affects metabolic consumption and muscular activation.

\section{DATA AVAILABILITY STATEMENT}

The datasets generated for this study are available on request to the corresponding author.

\section{REFERENCES}

Alkan, M. S., Gurocak, H., and Gonenc, B. (2013). Linear magnetorheological brake with serpentine flux path as a high force and low off-state friction actuator for haptics. J. Intell. Mater. Syst. Struct. 24, 1699-1713. doi: $10.1177 / 1045389$ X13484098

Awad, L. N., Bae, J., O'donnell, K., De Rossi, S. M., Hendron, K., Sloot, L. H., et al. (2017). A soft robotic exosuit improves walking in patients

\section{ETHICS STATEMENT}

The studies involving human participants were reviewed and approved by Comitato Etico Regione Liguria. The patients/participants provided their written informed consent to participate in this study. Written informed consent was obtained from the individual(s) for the publication of any identifiable images or data included in this article.

\section{AUTHOR CONTRIBUTIONS}

CD contribution on the hardware development, scientific study planning, and conduction of the experimental section and paper drafting. $\mathrm{AS}, \mathrm{AM}, \mathrm{EB}$, and $\mathrm{BH}$ contribution on the hardware development. AD and LO'S contribution on hardware development and proofreading. ER and KS contribution on the control aspects. BM and DC main review aspects. JO scientific review.

\section{FUNDING}

This work has received funding from the European Union's Horizon 2020 framework programme for research and innovation under grant agreement no. 688175 .

after stroke. Sci. Transl. Med. 9:eaai9084. doi: 10.1126/scitranslmed. aai9084

Brown, E., Rodenberg, N., Amend, J., Mozeika, A., Steltz, E., Zakin, M. R., et al. (2010). Universal robotic gripper based on the jamming of granular material. Proc. Natl. Acad. Sci. U.S.A. 107, 18809-18814. doi: 10.1073/pnas.1003 250107

Bureau, M., Keller, T., Perry, J., Velik, R., and Veneman, J. F. (2011). "Variable stiffness structure for limb attachment," in 2011 IEEE International 
Conference on Rehabilitation Robotics (ICORR) (Zurich: IEEE), 1-4. doi: 10.1109/ICORR.2011.5975350

Carlson, J. D., and Jolly, M. R. (2000). MR fluid, foam and elastomer devices. Mechatronics 10, 555-569. doi: 10.1016/S0957-4158(99)0 0064-1

Choi, I., Corson, N., Peiros, L., Hawkes, E. W., Keller, S., and Follmer, S. (2018). A soft, controllable, high force density linear brake utilizing layer jamming. IEEE Robot. Automat. Lett. 3, 450-457. doi: 10.1109/LRA.2017.2761938

Cooper, G., Sheret, I., McMillian, L., Siliverdis, K., Sha, N., Hodgins, D., et al. (2009). Inertial sensor-based knee flexion/extension angle estimation. J. Biomech. 42, 2678-2685. doi: 10.1016/j.jbiomech.2009.08.004

Dempster, W. T., and Gaughran, G. R. (1967). Properties of body segments based on size and weight. Am. J. Anat. 120, 33-54. doi: 10.1002/aja.1001200104

Di Natali, C., Poliero, T., Sposito, M., Graf, E., Bauer, C., Pauli, C., et al. (2019). Design and evaluation of a soft assistive lower limb exoskeleton. Robotica 37, 2014-2034. doi: 10.1017/S0263574719000067

Diller, S., Majidi, C., and Collins, S. H. (2016). “A lightweight, low-power electroadhesive clutch and spring for exoskeleton actuation," in 2016 IEEE International Conference on Robotics and Automation (ICRA) (Stockholm: IEEE), 682-689. doi: 10.1109/ICRA.2016.7487194

Endo, K., Paluska, D., and Herr, H. (2006). "A quasi-passive model of human leg function in level-ground walking," in 2006 IEEE/RSJ International Conference on Intelligent Robots and Systems (Beijing: IEEE), 4935-4939. doi: 10.1109/IROS.2006.282454

Farris, R. J., Quintero, H. A., and Goldfarb, M. (2011). Preliminary evaluation of a powered lower limb orthosis to aid walking in paraplegic individuals. IEEE Trans. Neural Syst. Rehabil. Eng. 19, 652-659. doi: 10.1109/TNSRE.2011.2163083

Follmer, S., Leithinger, D., Olwal, A., Cheng, N., and Ishii, H. (2012). “Jamming user interfaces: programmable particle stiffness and sensing for malleable and shape-changing devices," in Proceedings of the 25th Annual ACM Symposium on User Interface Software and Technology (Cambridge, MA: ACM), 519-528. doi: 10.1145/2380116.2380181

Graf, E. S., Bauer, C. M., Power, V., de Eyto, A., Bottenberg, E., Poliero, T., et al. (2018). "Basic functionality of a prototype wearable assistive soft exoskeleton for people with gait impairments: a case study," in Proceedings of the 11th PErvasive Technologies Related to Assistive Environments Conference (Corfu: ACM), 202-207. doi: 10.1145/3197768.3197779

Hauser, S., Robertson, M., Ijspeert, A., and Paik, J. (2017). Jammjoint: A variable stiffness device based on granular jamming for wearable joint support. IEEE Robot. Automat. Lett. 2, 849-855. doi: 10.1109/LRA.2017.2655109

Ikehara, T., Nagamura, K., Ushida, T., Tanaka, E., Saegusa, S., Kojima, S., et al. (2011). "Development of closed-fitting-type walking assistance device for legs and evaluation of muscle activity," in 2011 IEEE International Conference on Rehabilitation Robotics (ICORR) (Zurich: IEEE), 1-7. doi: 10.1109/ICORR.2011.5975449

Jezernik, S., Colombo, G., Keller, T., Frueh, H., and Morari, M. (2003). Robotic orthosis lokomat: a rehabilitation and research tool. Neuromodul. Technol. Neural Interface 6, 108-115. doi: 10.1046/j.1525-1403.2003.03017.x

Jiang, A., Xynogalas, G., Dasgupta, P., Althoefer, K., and Nanayakkara, T. (2012). "Design of a variable stiffness flexible manipulator with composite granular jamming and membrane coupling," in 2012 IEEE/RSJ International Conference on Intelligent Robots and Systems (IROS) (Vilamoura: IEEE), 2922-2927. doi: 10.1109/IROS.2012.6385696

Jin, S., Iwamoto, N., Hashimoto, K., and Yamamoto, M. (2017). Experimental evaluation of energy efficiency for a soft wearable robotic suit. IEEE Trans. Neural Syst. Rehabil. Eng. 25, 1192-1201. doi: 10.1109/TNSRE.2016.2613886

Kawamura, S., Kanaoka, K., Nakayama, Y., Jeon, J., and Fujimoto, D. (2003). "Improvement of passive elements for wearable haptic displays," in ICRA'03. IEEE International Conference on Robotics and Automation, 2003, Vol. 1 (Taipei: IEEE), 816-821. doi: 10.1109/ROBOT.2003.1241694

Kong, K., and Jeon, D. (2006). Design and control of an exoskeleton for the elderly and patients. IEEE/ASME Trans. Mechatron. 11, 428-432. doi: 10.1109/TMECH.2006.878550

Li, M., Ranzani, T., Sareh, S., Seneviratne, L. D., Dasgupta, P., Wurdemann, et al. (2014). Multi-fingered haptic palpation utilizing granular jamming stiffness feedback actuators. Smart Mater. Struct. 23:095007. doi: 10.1088/0964-1726/23/9/095007
Li, N., Yan, L., Qian, H., Wu, H., Wu, J., and Men, S. (2015). Review on lower extremity exoskeleton robot. Open Automat. Control Syst. J. 7, 441-453.

Manti, M., Cacucciolo, V., and Cianchetti, M. (2016). Stiffening in soft robotics: a review of the state of the art. IEEE Robot. Automat. Mag. 23, 93-106. doi: 10.1109/MRA.2016.2582718

Mecheels, J., and Umbach, K. H. (1977). The Psychrometric Range of Clothing Systems. Clothing Comfort. Michigan: Ann Arbor Science.

Mitsuda, T. (2017). "Variable-stiffness sheets obtained using fabric jamming and their applications in force displays," in World Haptics Conference (WHC) (Munich: IEEE), 364-369. doi: 10.1109/WHC.2017.7989929

Murray, S. A., Ha, K. H., and Goldfarb, M. (2014). "An assistive controller for a lower-limb exoskeleton for rehabilitation after stroke, and preliminary assessment thereof,' in Engineering in Medicine and Biology Society (EMBC), 2014 36th Annual International Conference of the IEEE (Chicago, IL: IEEE), 4083-4086. doi: 10.1109/EMBC.2014.6944521

Nakamura, T., Saito, K., and Kosuge, K. (2005). "Control of wearable walking support system based on human-model and GRF," in ICRA 2005. Proceedings of the 2005 IEEE International Conference on Robotics and Automation, 2005 (Barcelona: IEEE), 4394-4399. doi: 10.1109/ROBOT.2005.1570796

Nikitczuk, J., Weinberg, B., Canavan, P. K., and Mavroidis, C. (2010). Active knee rehabilitation orthotic device with variable damping characteristics implemented via an electrorheological fluid. IEEE/ASME Trans. Mechatron. 15, 952-960. doi: 10.1109/TMECH.2009.2036170

Oh, H.-U., and Onoda, J. (2002). An experimental study of a semiactive magnetorheological fluid variable damper for vibration suppression of truss structures. Smart Mater. Struct. 11:156. doi: 10.1088/0964-1726/11/1/318

Ortiz, J., Poliero, T., Cairoli, G., Graf, E., and Caldwell, D. G. (2018). Energy efficiency analysis and design optimization of an actuation system in a soft modular lower limb exoskeleton. IEEE Robot. Automat. Lett. 3, 484-491. doi: 10.1109/LRA.2017.2768119

Ortiz, J., Rocon, E., Power, V., de Eyto, A., O’Sullivan, L., Wirz, M., et al. (2017). "Xosoft-a vision for a soft modular lower limb exoskeleton," in Wearable Robotics: Challenges and Trends, eds J. González-Vargas, J. Ibáñez, J. Contreras-Vidal, H. van der Kooij, and J. Pons (Cham: Springer), 83-88. doi: 10.1007/978-3-319-46532-6_14

Petek, N. K. (1992). An Electronically Controlled Shock Absorber Using Electrorheological Fluid. Technical report, SAE Technical Paper, International Congress \& Exposition. doi: 10.4271/920275

Poliero, T., Di Natali, C., Sposito, M., Ortiz, J., Graf, E., Pauli, C., et al. (2018). "Soft wearable device for lower limb assistance: assessment of an optimized energy efficient actuation prototype," in 2018 IEEE International Conference on Soft Robotics (RoboSoft) (Livorno: IEEE), 559-564. doi: 10.1109/ROBOSOFT.2018.8405385

Power, V., O’Sullivan, L., de Eyto, A., Schülein, S., Nikamp, C., Bauer, C., et al. (2016). "Exploring user requirements for a lower body soft exoskeleton to assist mobility," in Proceedings of the 9th ACM International Conference on PErvasive Technologies Related to Assistive Environments (Corfu: ACM), 69. doi: 10.1145/2910674.2935827

Ramachandran, V., Shintake, J., and Floreano, D. (2019). All-fabric wearable electroadhesive clutch. Adv. Mat. Technol. 4:1800313. doi: 10.1002/admt.201800313

Sadeghi, A., Mondini, A., and Mazzolai, B. (2018). "Preliminary experimental study on variable stiffness structures based on textile jamming for wearable robotics," in International Symposium on Wearable Robotics (Pisa: Springer), 49-52. doi: 10.1007/978-3-030-01887-0_10

Sadeghi, A., Mondini, A., and Mazzolai, B. (2019a). "A vacuum powered soft textile-based clutch," in Actuators, Vol. 8, ed A. Miriyev, (Multidisciplinary Digital Publishing Institute), 47. doi: 10.3390/act8020047

Sadeghi, A., Mondini, A., Totaro, M., Mazzolai, B., and Beccai, L. (2019b). A wearable sensory textile-based clutch with high blocking force. Adv. Eng. Mat. 21:1900886. doi: 10.1002/adem.201900886

Sankai, Y. (2010). "Hal: hybrid assistive limb based on cybernics," in Robotics Research, eds M. Kaneko and Y. Nakamura (Hiroshima: Springer), 25-34. doi: 10.1007/978-3-642-14743-2_3

Sasaki, D., Noritsugu, T., and Takaiwa, M. (2013). "Development of pneumatic lower limb power assist wear driven with wearable air supply system," in 2013 IEEE/RSJ International Conference on Intelligent Robots and Systems (Tokyo: IEEE), 4440-4445. doi: 10.1109/IROS.2013.6696994 
Schmidt, K., Duarte, J. E., Grimmer, M., Sancho-Puchades, A., Wei, H., Easthope, C. S., et al. (2017). The myosuit: Bi-articular anti-gravity exosuit that reduces hip extensor activity in sitting transfers. Front. Neurorobot. 11:57. doi: 10.3389/fnbot.2017.00057

Sposito, M., Poliero, T., Di Natali, C., Ortiz, J., Pauli, C., Graf, E., et al. (2018). "Evaluation of xosoft beta-1 lower limb exoskeleton on a post stroke patient," in Sixth National Congress of Bioengineering (Milan).

Stanley, A. A., Gwilliam, J. C., and Okamura, A. M. (2013). "Haptic jamming: a deformable geometry, variable stiffness tactile display using pneumatics and particle jamming," in World Haptics Conference (WHC), 2013 (Daejeon: IEEE), 25-30. doi: 10.1109/WHC.2013.65 48379

Taghavi, M., Helps, T., Huang, B., and Rossiter, J. (2018). 3d-printed readyto-use variable-stiffness structures. IEEE Robot. Automat. Lett. 3, 2402-2407. doi: 10.1109/LRA.2018.2812917

Tonazzini, A., Shintake, J., Rognon, C., Ramachandran, V., Mintchev, S., and Floreano, D. (2018). "Variable stiffness strip with strain sensing for wearable robotics," in 2018 IEEE International Conference on Soft Robotics (RoboSoft) (Livorno: IEEE), 485-490. doi: 10.1109/ROBOSOFT.2018. 8405373

Totaro, M., Poliero, T., Mondini, A., Lucarotti, C., Cairoli, G., Ortiz, J., et al. (2017). Soft smart garments for lower limb joint position analysis. Sensors 17:2314. doi: $10.3390 /$ s17102314

Van Dijk, W., Van der Kooij, H., and Hekman, E. (2011). “A passive exoskeleton with artificial tendons: design and experimental evaluation,” in 2011 IEEE International Conference on Rehabilitation Robotics (ICORR) (Zurich: IEEE), 1-6. doi: 10.1109/ICORR.2011.5975470

Van Ham, R., Sugar, T. G., Vanderborght, B., Hollander, K. W., and Lefeber, D. (2009). Compliant actuator designs. IEEE Robot. Automat. Mag. 16, 81-94. doi: 10.1109/MRA.2009.933629
Veneman, J. F., Kruidhof, R., Hekman, E. E., Ekkelenkamp, R., Van Asseldonk, E. H., and Van Der Kooij, H. (2007). Design and evaluation of the lopes exoskeleton robot for interactive gait rehabilitation. IEEE Trans. Neural Syst. Rehabil. Eng. 15, 379-386. doi: 10.1109/TNSRE.2007.903919

Walsh, C. J., Endo, K., and Herr, H. (2007). A quasi-passive leg exoskeleton for load-carrying augmentation. Int. J. Human. Robot. 4, 487-506. doi: 10.1142/S0219843607001126

Wolf, S., Grioli, G., Eiberger, O., Friedl, W., Grebenstein, M., Höppner, H., et al. (2016). Variable stiffness actuators: Review on design and components. IEEE/ASME Trans. Mechatron. 21, 2418-2430. doi: 10.1109/TMECH.2015.2501019

Yan, T., Cempini, M., Oddo, C. M., and Vitiello, N. (2015). Review of assistive strategies in powered lower-limb orthoses and exoskeletons. Robot. Auton. Syst. 64, 120-136. doi: 10.1016/j.robot.2014.09.032

Zubrycki, I., and Granosik, G. (2017). Novel haptic device using jamming principle for providing kinaesthetic feedback in glove-based control interface. J. Intell. Robot. Syst. 85, 413-429. doi: 10.1007/s10846-016-0392-6

Conflict of Interest: The authors declare that the research was conducted in the absence of any commercial or financial relationships that could be construed as a potential conflict of interest.

Copyright (c) 2020 Di Natali, Sadeghi, Mondini, Bottenberg, Hartigan, De Eyto, O'Sullivan, Rocon, Stadler, Mazzolai, Caldwell and Ortiz. This is an open-access article distributed under the terms of the Creative Commons Attribution License (CC $B Y)$. The use, distribution or reproduction in other forums is permitted, provided the original author(s) and the copyright owner(s) are credited and that the original publication in this journal is cited, in accordance with accepted academic practice. No use, distribution or reproduction is permitted which does not comply with these terms. 